\title{
SISTEMA DE CALIFICACIÓN DE COSTOS REDITM UTILIZANDO LA METODOLOGÍA FEMA P-58, APLICADA A EDIFICACIONES DE MEDIANA Y GRAN ALTURA
}

\section{REDITM COST RATING SYSTEM USING THE FEM P. 58 APPLIED TO MEDIUM HEIGHT AND TALL BUILDINGS}

\author{
Roberto Aguiar, Cristhian Freire y Gustavo Yánez. \\ Departamento de Ciencias de la Tierra y la Construcción \\ Universidad de Fuerzas Armadas ESPE \\ Av. Gral. Rumiñahui s/n. Valle de los Chillos, Ecuador \\ rraguiar@espe.edu.ec \\ cpfreire@espe.edu.ec \\ gayanez@espe.edu.ec
}

\section{RESUMEN}

Este artículo estudia el sistema de calificación REDI ${ }^{\mathrm{TM}}$, que se basa en la calificación de costos estimados de reparación de edificaciones antes y después de eventos sísmicos.

La metodología FEMA P-58, permite usar el programa PACT, con el cual se evalúa el Bloque 3 de mediana altura del nuevo Centro de Investigaciones Científicas y de Post Grado de la Universidad de las Fuerzas Armadas ESPE, con los resultados del análisis sísmico espacial del método espectral con un sismo de diseño DBE, el análisis estático no lineal de la estructura, la curva de peligrosidad sísmica del sitio y curvas de fragilidad propuestas por PACT.

Para la edificación de gran altura, edificio Buzios afectado por el sismo de Manabí en Abril del 2016, se trabaja con las curvas de fragilidad de mampostería de PACT, para obtener el costo estimado de reparación, el mismo se compara con el costo real de reparación de mampostería y se validan las curvas de fragilidad que utiliza PACT, y de esta manera obtener la calificación de ambas edificaciones según el sistema de calificación de costos REDI ${ }^{\mathrm{TM}}$.

Palabras claves: REDI ${ }^{\mathrm{TM}}$ (Iniciativa de Diseño Sísmico Basada en la Resiliencia). FEMA (Agencia Federal para el Manejo de Emergencias). PACT (Herramienta de Cálculo para la Evaluación de Rendimiento). Curva de Peligrosidad Sísmica. Curvas de Fragilidad.

\section{ABSTRACT}

This paper studied the REDI ${ }^{\mathrm{TM}}$ rating system based on the categorization of estimated costs for repair before and after an earthquake. 
FEMA P-58 methodology allows using the PACT program, which Block 3 medium height of the new Center for Scientific Research and Graduate of the University of the ESPE Armed Forces is evaluated, with the results of spatial seismic analysis the spectral method with a design earthquake DBE, the nonlinear static analysis of the structure, the seismic hazard curve of the site and fragility curves proposed by PACT.

For the tall height, Buzios building was studied, which was affected by the earthquake produced in Manabí on April 2016, the curves of masonry fragility from PACT, to obtain the estimated repair cost comparing it to the real cost of the masonry repair and validating the fragility curves used by PACT. Thus, achieving the qualification of both structures according to the REDI ${ }^{\mathrm{TM}}$ cost rating system.

Keywords: REDI ${ }^{\mathrm{TM}}$ (Resilience-based Earthquake Design Initiative). FEMA (Federal Emergency Management Agency). PACT (Performance Assessment Calculation Tool). Seismic Hazard Curve. Fragility Curves.

\section{INTRODUCCIÓN}

\subsection{Sistema de calificación REDi ${ }^{\mathrm{TM}}$}

(Almufti - Willford, 2013). En los códigos de construcción actuales no se considera la capacidad de resiliencia sísmica de una comunidad para recuperarse rápidamente después de un sismo de gran magnitud. El objetivo del código mediante un diseño eficiente de la estructura es proteger la vida de los ocupantes del edificio; y aún si la estructura cumple el objetivo del código está expuesta a daños significativos en la estructura del edificio, componentes arquitectónicos, fachadas, equipamiento mecánico/eléctrico/fontanería (MEP), y contenidos de la construcción.

En consecuencia, no es sorprendente que cuando un sismo de gran magnitud golpea una ciudad las pérdidas por daños en las infraestructuras de los edificios son muy grandes en términos económicos. Las pérdidas directas incluyen: los costos financieros de demolición, reparación y restauración de los servicios públicos post-terremoto.

Los objetivos de diseño y preparación de la sociedad moderna para un terremoto deben mejorarse, ya que actualmente es posible lograr una mayor resistencia con una inversión adicional mínima, comparada con el costo total del proyecto. El Sistema de calificación REDi ${ }^{\mathrm{TM}}$ considera: planificación de resiliencia, resiliencia del edificio, ambiente de resiliencia y evaluación del tiempo de inactividad; para este estudio únicamente se califica la resiliencia del edificio, en base a costos de reparación y derivas máximas.

Las expectativas para los Códigos de Diseño de los Edificios evolucionan durante los años en respuesta a la observación de los daños en terremotos reales y puesta en práctica de programas de investigación. El objetivo durante la historia es proporcionar "seguridad de vida" con "terremotos de diseño", pero los resultados de dichos programas de investigación no son cuantificados hasta 
recientemente, y este detalle todavía no entienden los propietarios de los edificios y sus ocupantes.

\subsection{Evaluación de Pérdidas}

(Almufti - Willford, 2013). El enfoque de diseño basado en el cumplimiento de los objetivos de capacidad de recuperación $R E D i^{T M}$ se mide a través de la evaluación de pérdidas que cuantifican el riesgo de terremotos en términos de pérdidas económicas directas.

Para la evaluación de pérdidas financieras de edificaciones se utiliza el programa PACT, una herramienta propuesta por FEMA que permite al usuario definir la cantidad y la ubicación de todos los componentes de un edificio y su contenido. Las respuestas inducidas por terremotos (derivas, aceleraciones, etc.) de la estructura son primero predichas por un modelo de cálculo, como el modal espectral. Los daños previstos para cada uno de los componentes de la construcción causados por el terremoto se calculan en función de las curvas de fragilidad y los parámetros de demanda. Finalmente, las consecuencias se cuantifican en términos de costos estimados de reparación, y el riesgo de controladores (aquellos componentes que causan la mayor parte de las pérdidas) se identifican.

Tabla 1 Objetivos de categorización REDI TM (Almufti - Willford, 2013).

\begin{tabular}{|c|c|c|l|}
\hline Categorías & $\begin{array}{c}\text { Pérdida Financiera } \\
\text { Directa }\end{array}$ & $\begin{array}{c}\text { Deriva } \\
\text { Máxima } \\
\text { Requerida }\end{array}$ & Seguridad de los Ocupantes \\
\hline PLATINO & $<2,5 \%$ Costo Total & $<0,50 \%$ & $\begin{array}{l}\text { Lesiones físicas debido a la } \\
\text { falla de piezas no expuestas de } \\
\text { construcción }\end{array}$ \\
\hline ORO & $<5 \%$ Costo Total & $<0,50 \%$ & $\begin{array}{l}\text { Lesiones físicas debido a la } \\
\text { falla de piezas no expuestas de } \\
\text { construcción }\end{array}$ \\
\hline PLATA & $<10 \%$ Costo Total & $<0,50 \%$ & $\begin{array}{l}\text { Lesión física puede ocurrir a } \\
\text { partir de la caída de } \\
\text { componentes (pero no colapso } \\
\text { estructural), las muertes son } \\
\text { poco probables }\end{array}$ \\
\hline
\end{tabular}

\section{MANUAL DE USUARIO DEL PROGRAMA PACT}

El programa PACT (Herramienta de Cálculo para la Evaluación del Rendimiento) es una herramienta de cálculo que sirve para evaluar el rendimiento sísmico, es decir, porcentajes de daños que se obtienen ante fuerzas sísmicas de edificios ya existentes o que se van a construir, dicho programa está en el Volumen 3 del FEMA P-58-3 conjuntamente con el Volumen 1 y 2 que contienen la 
Metodología e Implementación para la evaluación por rendimiento sísmico. El programa PACT considera para modelar, un edificio con elementos estructurales y no estructurales. El resultado que se obtiene al final de la evaluación por rendimiento es el costo estimado de reparación.

\subsection{Instalación}

El instalador del programa de evaluación por rendimiento sísmico PACT se encuentra en el Volumen 3 del FEMA P-58. La instalación es totalmente sencilla y en pocos minutos el programa PACT estará instalado y listo para su funcionamiento.

\subsection{Panel del control del PACT}

Al abrir el programa se puede observar la siguiente ventana:

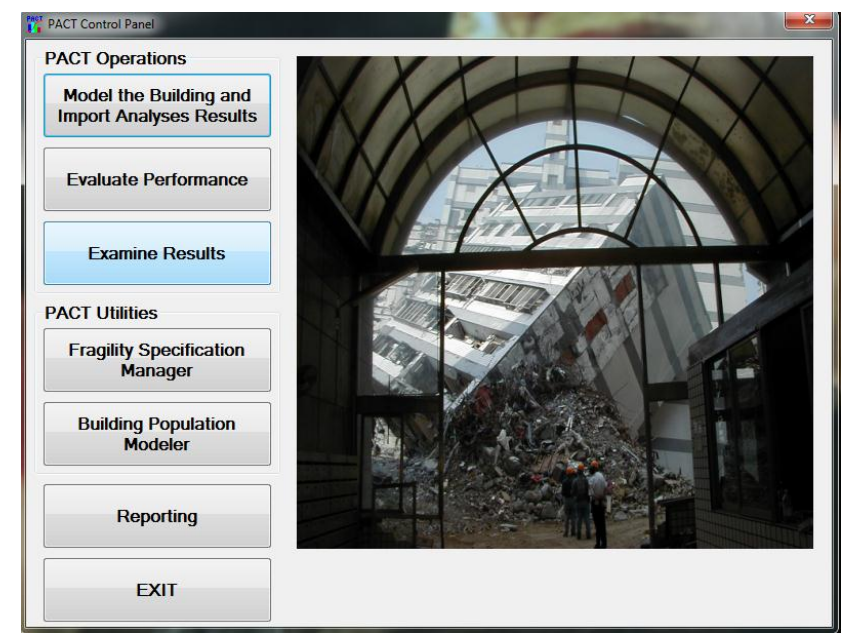

Figura 1 Panel de control (FEMA P-58 VOL 3, 2012)

\subsubsection{Operaciones de PACT}

Modelar el Edificio e Importar los Resultados del Análisis.- en este botón se puede crear un nuevo modelo y también abrir un modelo ya creado para realizar modificaciones en caso de ser necesario.

Evaluación del Rendimiento.- sirve para correr el modelo.

Examinar resultados.- revisión de resultados.

\subsubsection{Utilidades de PACT}

Especificaciones de Fragilidad.- en este ítem se tiene las especificaciones de fragilidad de los elementos estructurales y no estructurales.

Población del edificio.- en este ítem se tiene los porcentajes de ocupación en el edificio según la hora. 
Presentación de informes.- este ítem permite cambiar la ubicación donde se guardan los archivos en PACT.

Salir.- para cerrar el programa.

\subsection{Generación de un Modelo}

Para realizar la generación de un nuevo modelo se da clic en el botón "Modelo de Edificio e Importar Resultados del Análisis Estructural" de la Figura 1, y se despliega la barra de herramientas con la primera pestaña, Información del proyecto.

\subsubsection{Información del Proyecto}

Antes de empezar en la construcción del modelo es necesario seleccionar las unidades en la pestaña TOOLS $\rightarrow$ Options $\rightarrow$ Metric de la barra de herramientas, por ser las unidades internacionales (SI) y que se trabajan en Ecuador.

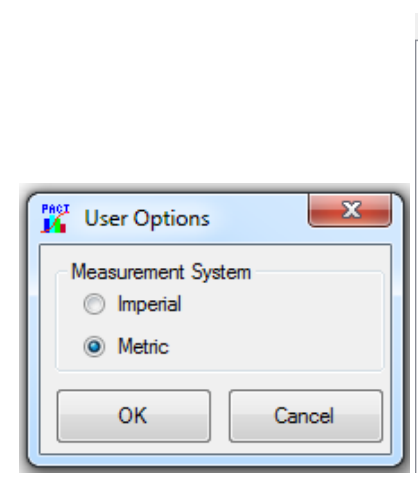

a)

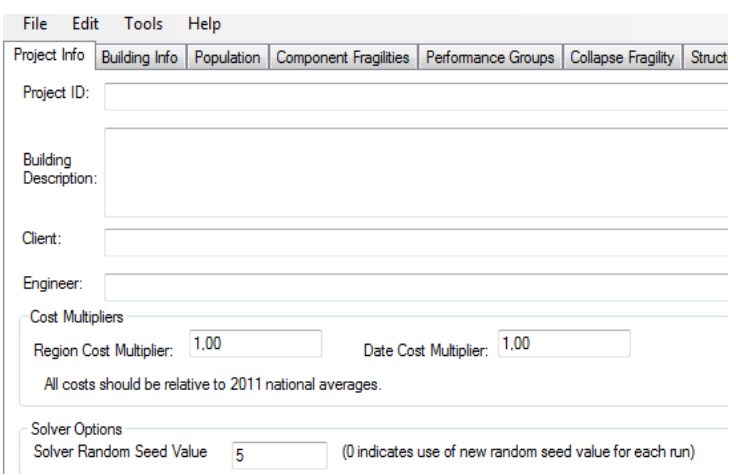

b)

Figura 2 a) Sistemas de Medida b) Información del Proyecto (FEMA P-58

VOL 3, 2012)

En esta pestaña se tiene:

- Nombre del proyecto

- Descripción del edificio

- Cliente

- Ingeniero responsable

- Factores predeterminados relacionados a costos según la región y fecha. Los costos son referentes a promedios del estado de Los Angeles (California), año 2011 (FEMA P-58 VOL 2, 2012). Para esta investigación se realiza una comparación entre los costos de California del 2011 y los costos actuales del Ecuador, de la cual se obtiene un incremento del $70 \%$, cabe recalcar que no se puede modificar la codificación del programa sin una previa autorización de los autores.

PACT utiliza los campos multiplicadores de costos según la región y la fecha para ajustar los costos de reparación de componentes suministrados a costos actuales. Los usuarios pueden tomar la escala y variación de los costos regionales a través de la entrada del multiplicador de costos, que utiliza precios de 
construcción de la región. El multiplicador de costos sólo se aplica a los datos de los costos previstos en el PACT. Si el usuario introduce de forma independiente los costos de reparación de los elementos de todos los grupos de rendimiento de la evaluación, los multiplicadores de costos de la región y la fecha se deben ingresar con un valor de 1,0 .

- Valor de origen de soluciones aleatorias

El valor de origen genera el número de secuencias aleatorias que usa internamente el programa para la evaluación del desempeño. Dicho valor debe ser diferente de cero para realizar varias evaluaciones de un mismo modelo, caso contrario se obtienen resultados diferentes.

\subsubsection{Información del Edificio}

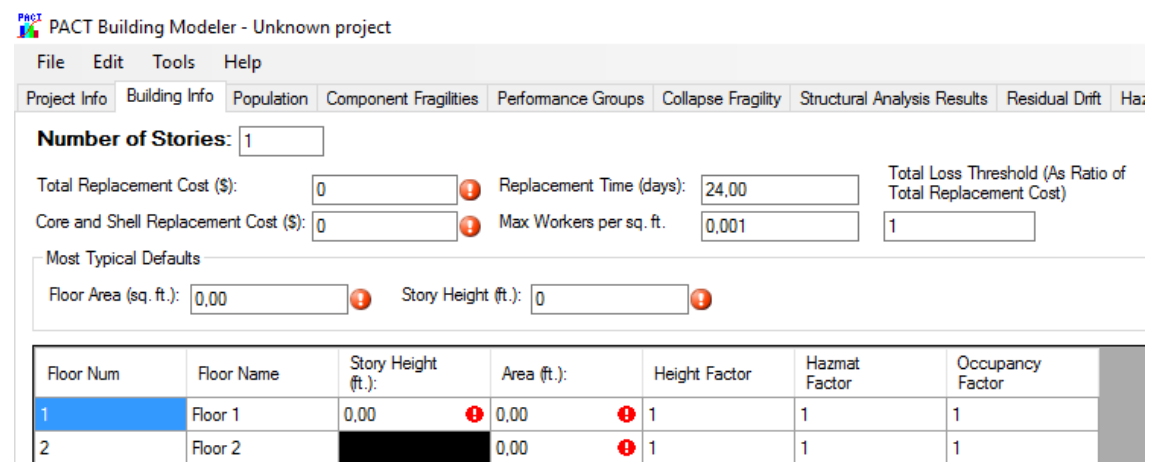

Figura 3 Información del Edificio (FEMA P-58 VOL 3, 2012)

- Costo total del edificio con acabados

- Duración de la construcción del edificio (días).

- Costo del edificio sin acabados.

- Trabajadores existentes por metros cuadrados (FEMA recomienda valor que está predeterminado).

PACT proporciona un valor predeterminado de 0.001 , que corresponde a 1 trabajador por cada $1000 \mathrm{ft}^{2}$ de superficie y en el caso de esta investigación corresponde a 1 trabajador por cada $92.9 \mathrm{~m}^{2}$

- Umbral de pérdida total

PACT utiliza un valor predeterminado de 1 para maximizar la cantidad de información de los resultados que se obtendrán en una evaluación. 
- Número de pisos, área y altura de entrepiso.

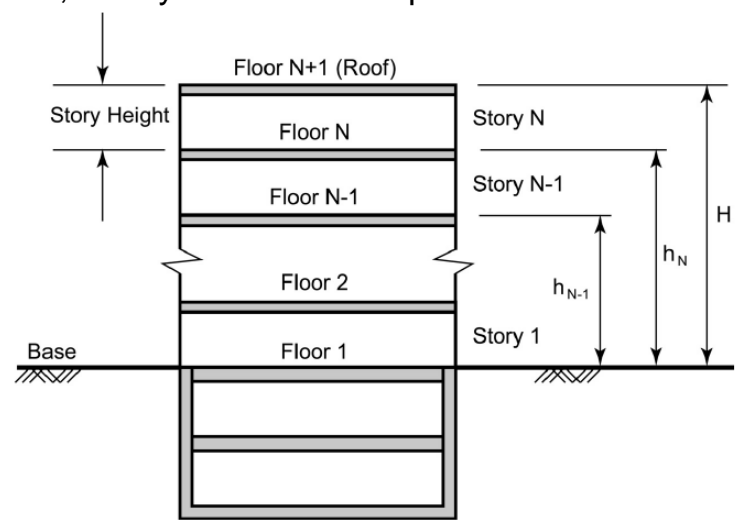

Figura 4 Interpretación de los pisos (FEMA P-58 VOL 2, 2012)

- Matriz con factores: por altura, materiales peligrosos y ocupación.

Tabla 2 Factores por altura (FEMA P-58 VOL 2, 2012)

\begin{tabular}{|l|c|}
\hline \multicolumn{1}{|c|}{$\begin{array}{c}\text { Niveles del } \\
\text { Edificio }\end{array}$} & $\begin{array}{c}\text { Factor por } \\
\text { Altura }\end{array}$ \\
\hline De 1 a 4 Pisos & 1,00 \\
\hline De 5 a 10 Pisos & 1,08 \\
\hline $\begin{array}{l}11 \text { pisos y más } \\
\text { altos }\end{array}$ & 1,16 \\
\hline
\end{tabular}

(FEMA P-58 VOL 2, 2012). A menos que se especifique información, se toma un factor de 1,0 para materiales peligrosos. El rango del factor por materiales peligrosos va desde 1,00 para edificios modernos con contenido de materiales peligrosos insignificante, y hasta 1,20 para los edificios que contienen cantidades significativas de materiales peligrosos, que incluye pintura a base de plomo y asbesto.

Tabla 3 Factores por ocupación (FEMA P-58 VOL 2, 2012)

\begin{tabular}{|l|c|c|}
\hline Categoría de Ocupación & Desocupado & Ocupado \\
\hline Oficinas de comercio & 1,00 & 1,20 \\
\hline Centro de Investigaciones & 1,40 & 1,80 \\
\hline Cuidado de Salud & 1,50 & 2,00 \\
\hline Educación & 1,00 & 1,10 \\
\hline Residencial & 1,10 & 1,20 \\
\hline Venta al por menor & 1,20 & 1,30 \\
\hline Bodega & 1,10 & 1,10 \\
\hline Hotelería & 1,10 & 1,10 \\
\hline
\end{tabular}




\subsubsection{Población del Edificio}

En esta ventana se selecciona la población que va a ocupar el edificio en un futuro o que está siendo ocupada si es un edificio ya existente.

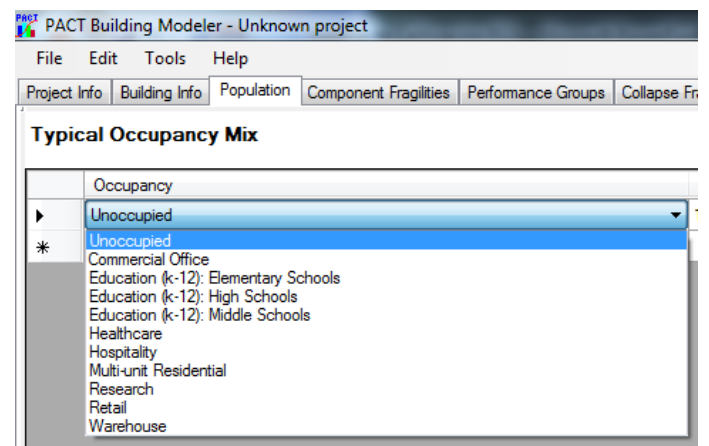

Figura 5 Tipos de ocupación (FEMA P-58 VOL 3, 2012)

El edificio puede estar desocupado o tener varios tipos de ocupación, los cuales se enlistan a continuación:

- Oficinas comerciales

- Educación (escuelas, colegios, universidades)

- Cuidados de salud

- Hotelería

- Residencial

- $\quad$ Centro de investigaciones

- Venta al por menor

- Bodegas

Los porcentajes de ocupación de cada día, semana y mes están predeterminados según el tipo de ocupación que se seleccione.

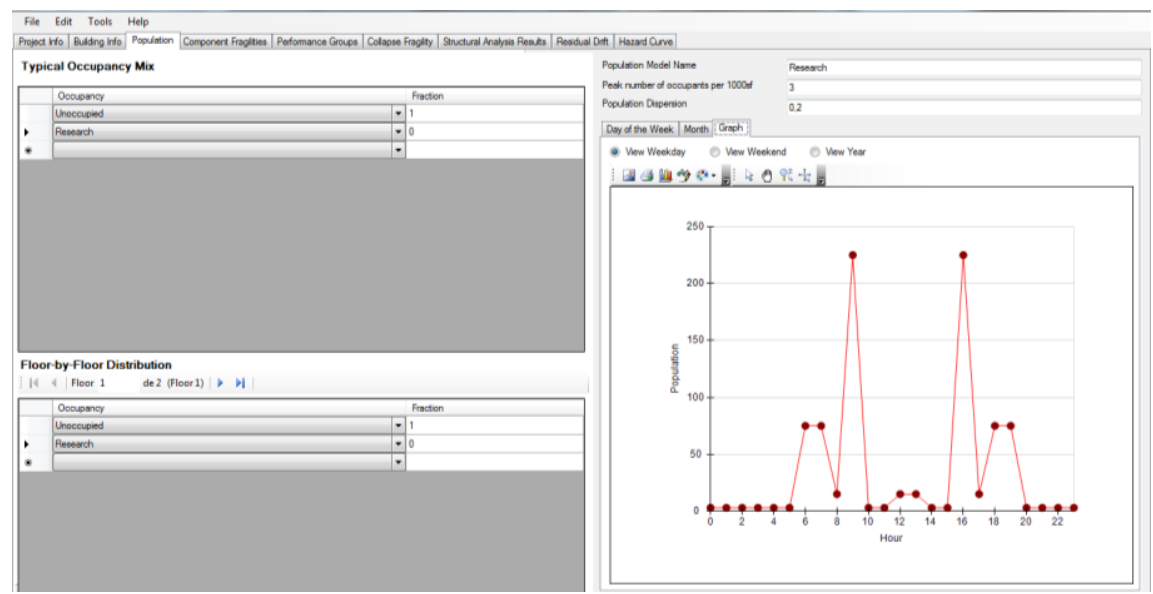

Figura 6 Población (FEMA P-58 VOL 3, 2012) 


\subsubsection{Componentes de Fragilidad}

Se deben seleccionar los elementos estructurales y no estructurales que se tiene en el edificio. PACT proporciona una base de datos de elementos estructurales y no estructurales, según las necesidades se elegirán las tipologías. A continuación se presenta una descripción macro de las tipologías previstas:

- A - Subestructura

- B - Estructura

- $\quad$ C - Interiores

- D - Servicios

- $\quad E-$ Equipamiento inmobiliario

- $\quad F-$ Construcciones especiales y demolición

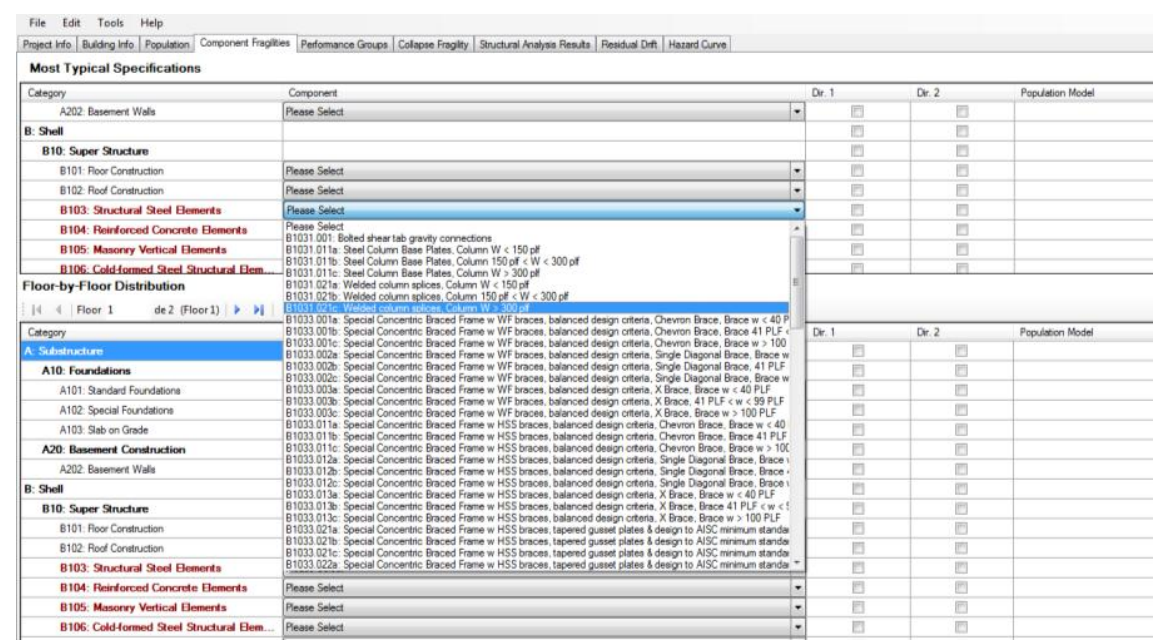

Figura 7 Componentes de fragilidad (FEMA P-58 VOL 3, 2012)

Cabe recalcar que la base de datos se identifica por códigos según el tipo de elemento; realizar una adecuada selección de los elementos estructurales y no estructurales influye notablemente en el rendimiento sísmico del edificio.

\subsubsection{Grupos de Rendimiento}

Grupos de rendimiento se denomina a todos los elementos (estructurales y no estructurales) que actúan en un piso, tanto en dirección 1 (Norte-Sur), dirección 2 (Este-Oeste) y sin dirección.

En esta ventana se añaden todos los elementos (estructurales y no estructurales) por cada piso y dirección; y colocar la cantidad de elementos y su dispersión. Depende del criterio del ingeniero evaluador si considera que un elemento es frágil o no. 


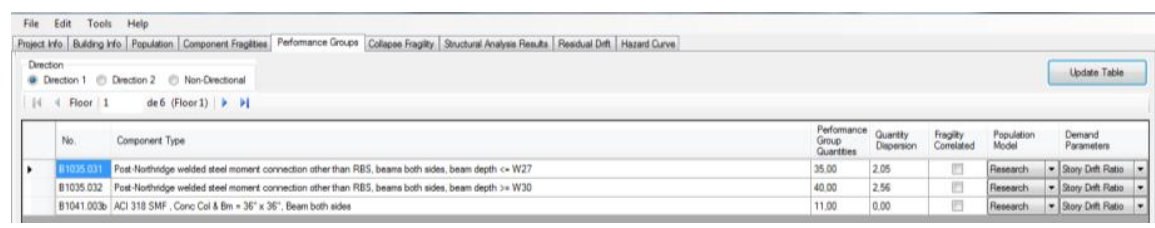

Figura 8 Grupos de rendimiento (FEMA P-58 VOL 3, 2012)

El botón "Update Table" sirve para cargar todos los elementos que se seleccionan anteriormente en cada piso y dirección. Es importante conocer que el parámetro de demanda se selecciona automáticamente por cada elemento.

Ejemplo de un grupo de fragilidad de un marco de acero de dos pisos en un edificio de oficinas:

Tabla 4 Grupo de fragilidad (FEMA P-58 VOL 2, 2012)

\begin{tabular}{|c|c|}
\hline Descripción & $\begin{array}{l}\text { Parámetros de } \\
\text { demanda }\end{array}$ \\
\hline $\begin{array}{l}\text { Conexiones de acero } \\
\text { estructural }\end{array}$ & Deriva \\
\hline $\begin{array}{l}\text { Muros de corte } \\
\text { exteriores }\end{array}$ & Deriva \\
\hline $\begin{array}{l}\text { Paredes interiores, } \\
\text { particiones }\end{array}$ & Deriva \\
\hline Techos suspendidos & Aceleración de piso \\
\hline Ascensor de tracción & $\begin{array}{l}\text { Máxima aceleración } \\
\text { del suelo }\end{array}$ \\
\hline Enfriadores & Aceleración de piso \\
\hline Modulares de oficina & Aceleración de piso \\
\hline Archivadores & Velocidad de piso \\
\hline
\end{tabular}

\subsubsection{Fragilidad de Colapso}

En esta pestaña se debe ingresar la Aceleración de Colapso en función de la gravedad "Sa(g)" y su dispersión. A continuación se deben colocar los modos de colapso que se esperan en el edificio. 


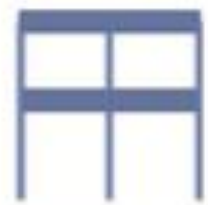

Undamaged

Structure

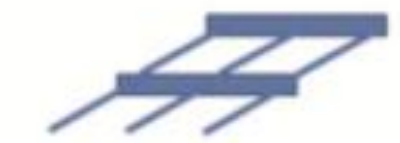

Collapse Mode 3

Multi-story mechanism
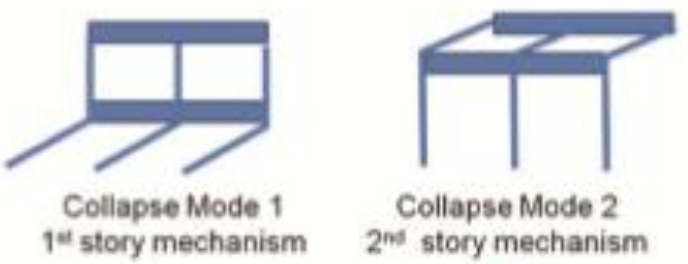

Collapse Mode 2

$2^{\text {ms }}$ story mechanism

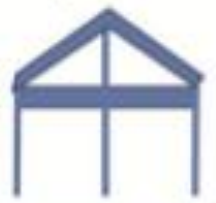

Collapse Mode 4

$2^{\text {nd }}$ story column shear failuer

Figura 9 Modos potenciales de colapso (FEMA P-58 VOL 2, 2012)

(FEMA P-58 VOL 2, 2012). Para cada modo potencial del colapso, estimar una probabilidad de ocurrencia que supone modos de colapso mutuamente excluyentes, es decir, las probabilidades de todos los modos deben sumar un valor de 1,0 .

(FEMA P-58 VOL 2, 2012). En la figura 9, el modo de colapso 1 es el colapso del primer piso, en el que se comprime el 100\% de la primera superficie de suelo, por un posible efecto de piso blando, pero nada del resto de pisos. Modo de colapso 2 es el colapso del segundo piso en el que se comprime el $100 \%$ del área del segundo piso, por un posible efecto de piso blando, pero nada de los demás pisos. Modo de colapso 3 es un colapso total en el que el $100 \%$ de las áreas de todos pisos colapsan. Modo de colapso 4 es un colapso parcial de la segunda planta, en la que según los autores del FEMA P-58 el $40 \%$ de la superficie del segundo piso está sujeto a la compresión.

La fracción de escombros en cada piso se coloca en función de los modos potenciales de colapso previamente seleccionados, con su respectivo porcentaje de ocurrencia.

(FEMA P-58 VOL 2, 2012). PACT requiere la definición de la Tasa media de fatalidad (COV y asociados) y la tasa media de lesiones (COV y asociados). La Tasa media de fatalidad es la fracción de ocupantes que habitan en la zona afectada, y que están fatalmente heridos por el colapso. Los usuarios del programa PACT deben seleccionar los valores según su criterio, y considerar los tipos de desechos que pueden originarse con ciertos tipos de construcciones y su letalidad. Se requiere un juicio similar para la tasa media de lesiones. Estos dos valores (tasa media de fatalidad y lesiones) deben sumar a un valor de 1,0. 


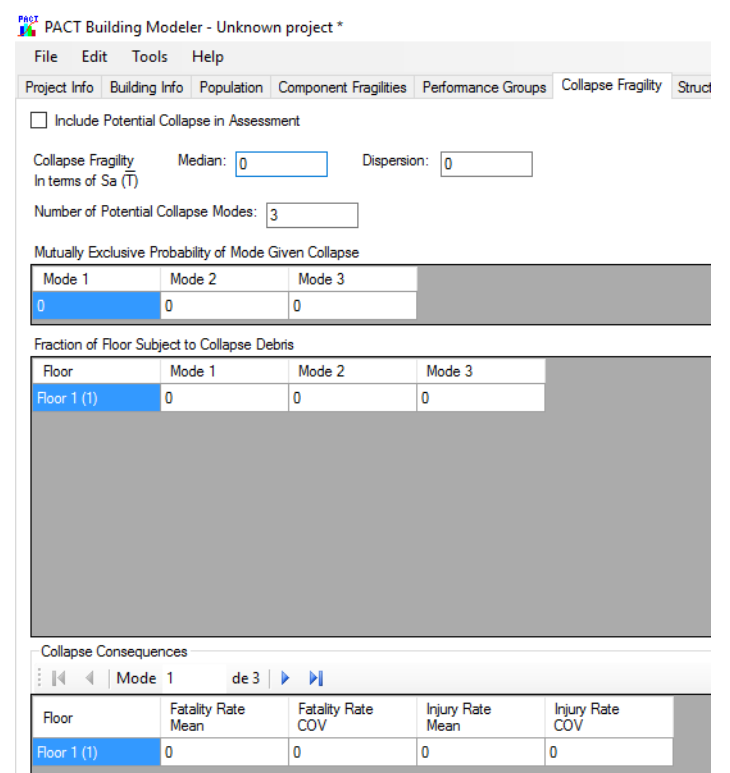

Figura 10 Fragilidad de colapso (FEMA P-58 VOL 3, 2012)

La fragilidad de colapso se puede excluir de la evaluación, si no se cuenta con un registro de datos acerca de la tasa media de fatalidad y tasa media de lesiones, que sirva de guía para tener un criterio de cuales valores se ingresan en la matriz de consecuencias de colapso, puesto que son valores que dependen del juicio de expertos.

\subsubsection{Resultados del análisis estructural}

El resultado del análisis estructural proviene de la realización de un modelo matemático del edificio, el mismo que puede ser evaluado con el método lineal o no lineal, para conseguir las propiedades dinámicas de la estructura, y posteriormente obtener derivas, aceleraciones y velocidades; que serán parámetros de demanda en la evaluación por rendimiento.

Se debe seleccionar el tipo de evaluación, tipo de análisis e ingresar el número típico de vectores de demanda y número de realizaciones. El número típico de vectores de demanda se refiere al sismo de diseño que se considera y el número de realizaciones son las interacciones de las secuencias que el programa realiza hasta obtener un resultado.

\section{La direccionalidad de la demanda}

(FEMA P-58 VOL 2, 2012). Los usuarios del programa PACT deben introducir derivas, la aceleraciones y velocidades; por piso y en cada dirección. PACT calcula la relación de deriva, aceleración y velocidad no direccional, como 1,2 veces el máximo de los dos valores obtenidos en cada piso a lo largo de los dos ejes horizontales. 
Se deben añadir las intensidades necesarias con las que se trabaja en la curva de peligrosidad sísmica, que se ingresa posteriormente; FEMA trabaja con un rango de 8 intensidades (Véase FEMA P-58 Vol, 2 Cap 3).

Como paso final en esta pestaña se deben ingresar los resultados numéricos de los parámetros de demanda (derivas, aceleraciones o velocidades) y sus dispersiones para cada intensidad.

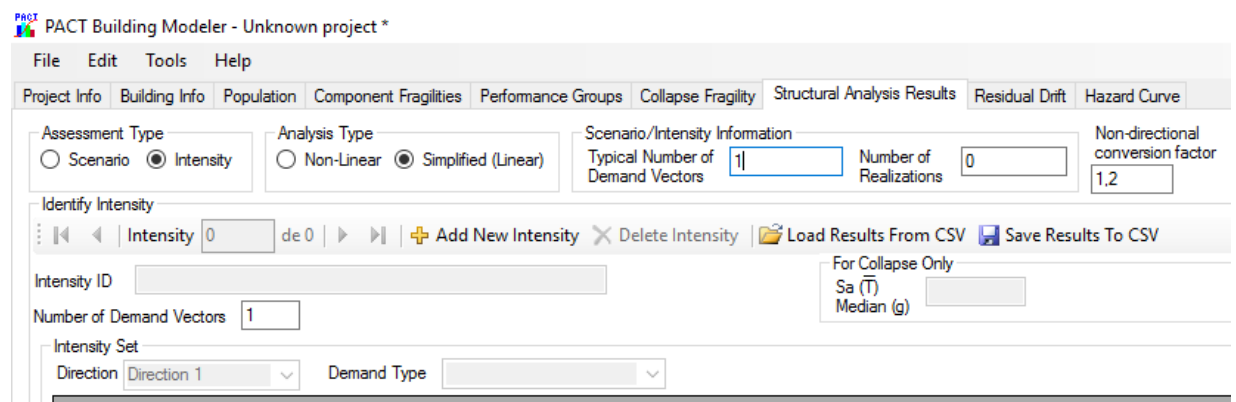

Figura 11 Resultados del análisis estructural (FEMA P-58 VOL 3, 2012)

\subsubsection{Deriva Residual}

La deriva residual es aquella que se obtiene una vez que la estructura se afecta por el sismo. Se calcula con los desplazamientos medidos desde el centro de gravedad del piso en cada dirección que se desplaza.

PACT proporciona una función de fragilidad asumida para la relación de la deriva residual que utiliza un valor de media irreparable de $1 \%$ de deriva residual y una dispersión de 0,30.

Si se desea incluir el uso de derivas residuales calculadas en el análisis, se debe colocar la deriva residual calculada con su dispersión.

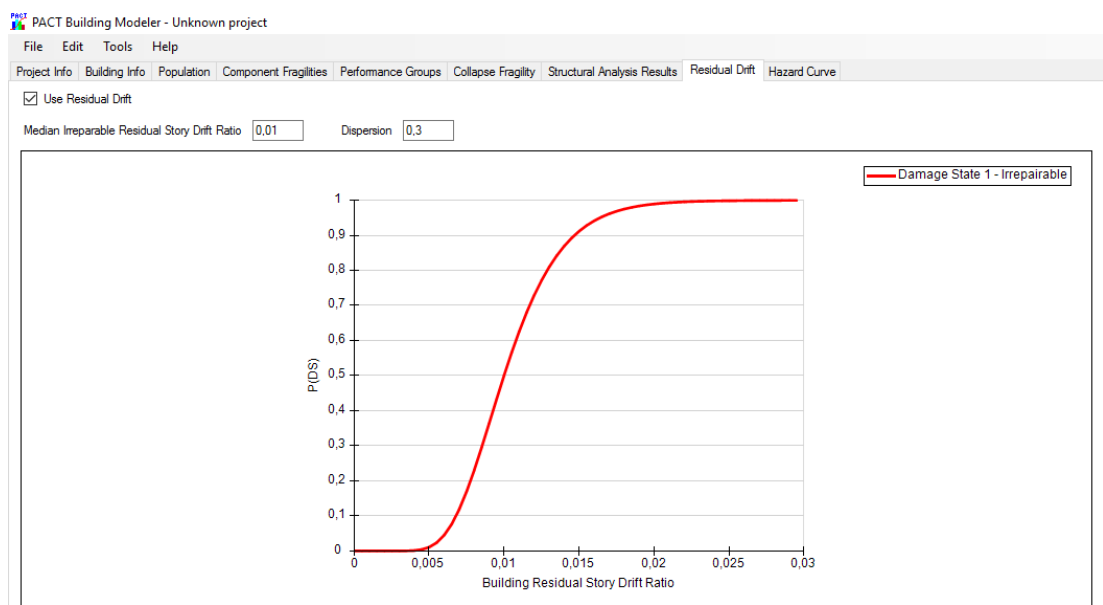

Figura 12 Deriva residual (FEMA P-58 VOL 3, 2012) 


\subsubsection{Curva de peligrosidad}

La curva de peligrosidad sísmica de la zona se debe ingresar según las características del sitio, que implica: zona sísmica, tipo de suelo y tasa anual de recurrencia.

PACT recomienda utilizar 8 valores de aceleraciones con sus respectivas tasas anuales de recurrencia, lo cual sirve para generar la curva de peligrosidad sísmica de la zona donde se ubica el edificio en análisis.

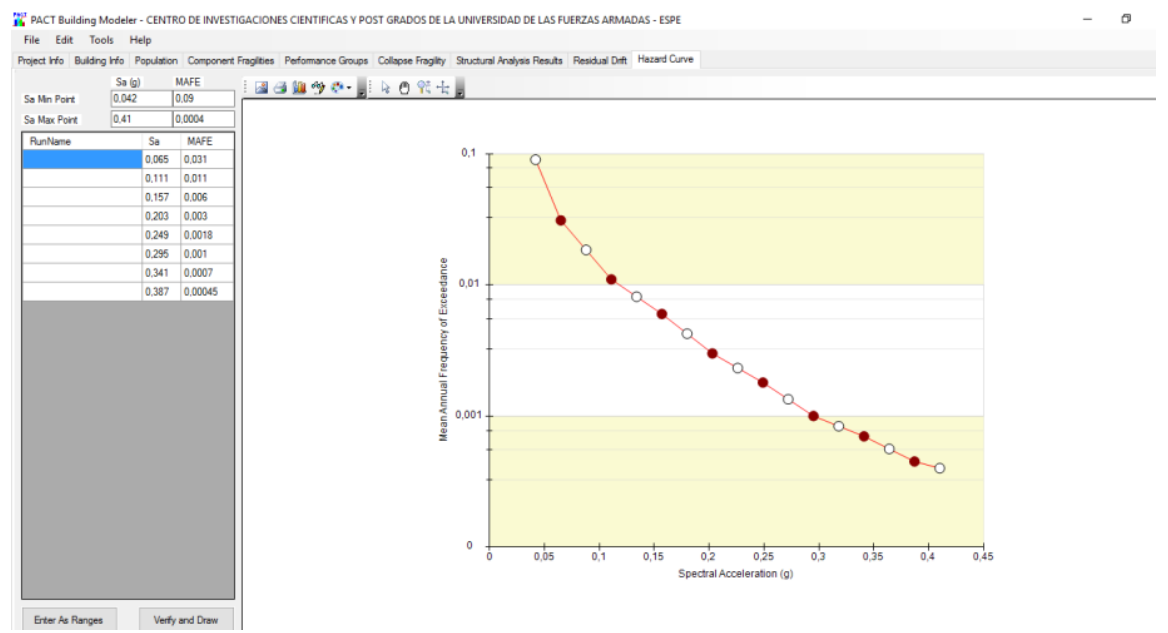

Figura 13 Curva de peligrosidad (FEMA P-58 VOL 3, 2012)

Con la curva de peligrosidad sísmica se evaluan todos los datos del edificio ingresados anteriormente, con lo cual se obtiene el costo de reparación estimado.

\section{APLICACIÓN DEL PACT EN EL BLOQUE 3 DEL NUEVO CENTRO DE INVESTIGACIONES CIENTÍFICAS Y DE POST GRADO DE LA UNIVERSIDAD DE LAS FUERZAS ARMADAS ESPE}

En el Proyecto del nuevo Centro de Investigaciones Científicas y de Post Grado de la Universidad de Fuerzas Armadas ESPE, se tiene el bloque estructural identificado como 3, el cual es objeto de este análisis.

Se utilizan aisladores sísmicos en todos los ejes de columnas, los que se colocan en el primer piso al nivel +4.04 , los aisladores están sobre columnas de hormigón armado de dimensiones $0.80 \times 0.80 \mathrm{~m}$, ya que en el subsuelo de este bloque se colocarán equipos muy sensibles a vibraciones para investigaciones en nanotecnología. Cabe recalcar que para el análisis se realiza la evaluación de la estructura con y sin aisladores. 


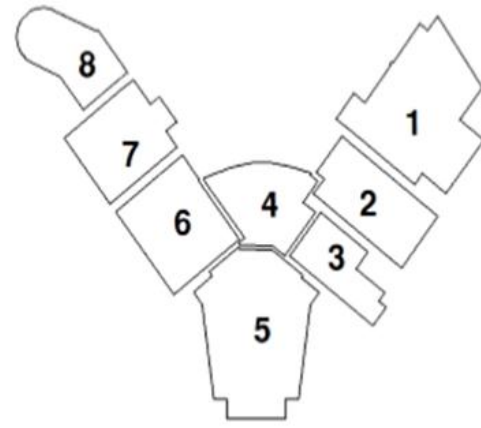

a)

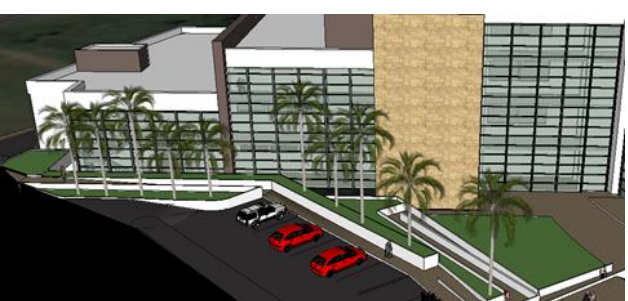

b)

Figura 14 a) Distribución de los bloques b) Proyecto arquitectónico (Aguiar y Pazmiño, 2016)

\subsection{Evaluación del edificio de mediana altura en PACT}

\subsubsection{Descripción del Bloque 3}

El Bloque 3, consta de 5 pisos; en el primer piso se tienen columnas de hormigón armado de $0.80 \times 0.80 \mathrm{~m}$ con vigas de acero tipo I de $550 \times 300 \times 25 \times 13$ $\mathrm{mm}$. Sobre las columnas del primer piso se colocan los aisladores triple péndulo de fricción. En los demás pisos se cuenta con columnas de acero tubulares de

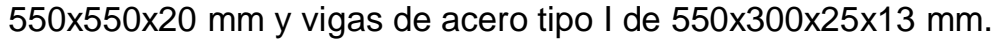

Tabla 5 Secciones de los elementos estructurales

\begin{tabular}{|c|c|c|}
\hline $\begin{array}{c}\text { Sección de } \\
\text { Columnas de H. A. }\end{array}$ & $\begin{array}{c}\text { Sección de } \\
\text { Columnas de Acero } \\
\text { Tubulares }\end{array}$ & $\begin{array}{c}\text { Sección de Vigas de } \\
\text { Acero Tipo I }\end{array}$ \\
\hline & & \\
\hline
\end{tabular}

A continuación se presenta las vistas en planta correspondientes al Bloque 3. En la parte izquierda se tienen los niveles: $0+00,+4.94,+9.88$ y +14.82 de losas de pisos intermedios, a la derecha se tiene la losa de cubierta en el nivel +19.76 . 


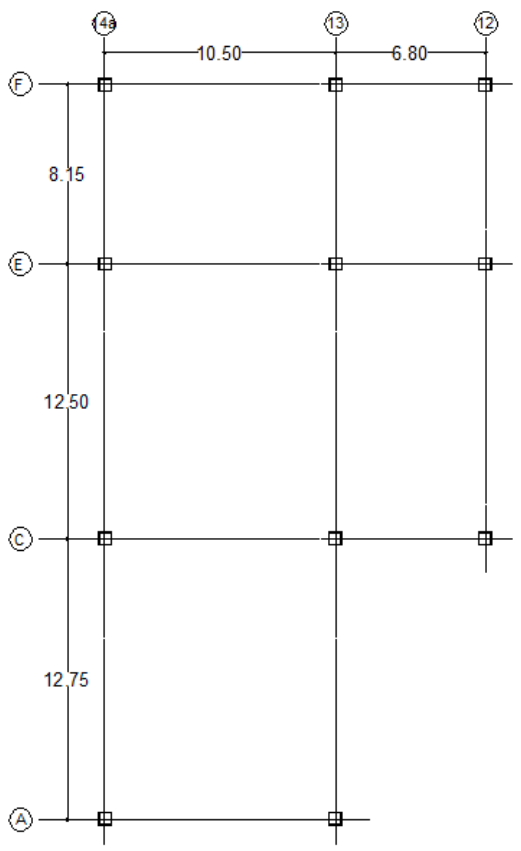

a)

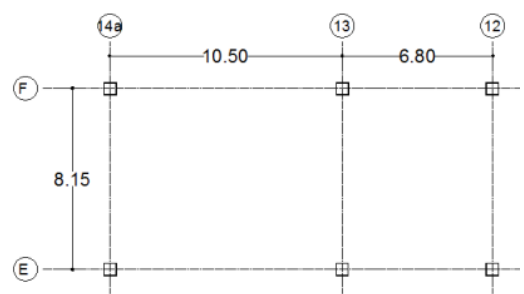

b)

Figura 15 a) Losas $+0.00+4.94+9.88+14.82$ b) Losa +19.76

Además se tienen cortes longitudinales y vista tridimensional del bloque 3 , en las que se aprecian los aisladores, las columnas de hormigón armado, las columnas y vigas de acero de la estructura del bloque 3 .

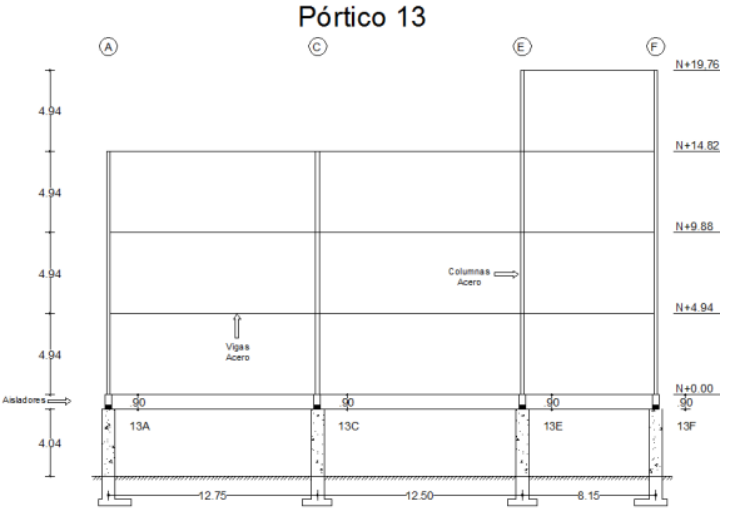

a)

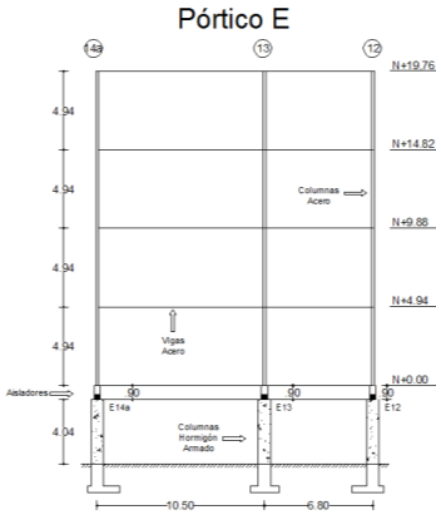

b)

Figura 16 Vistas en elevación a) Pórtico 13 b) Pórtico E 


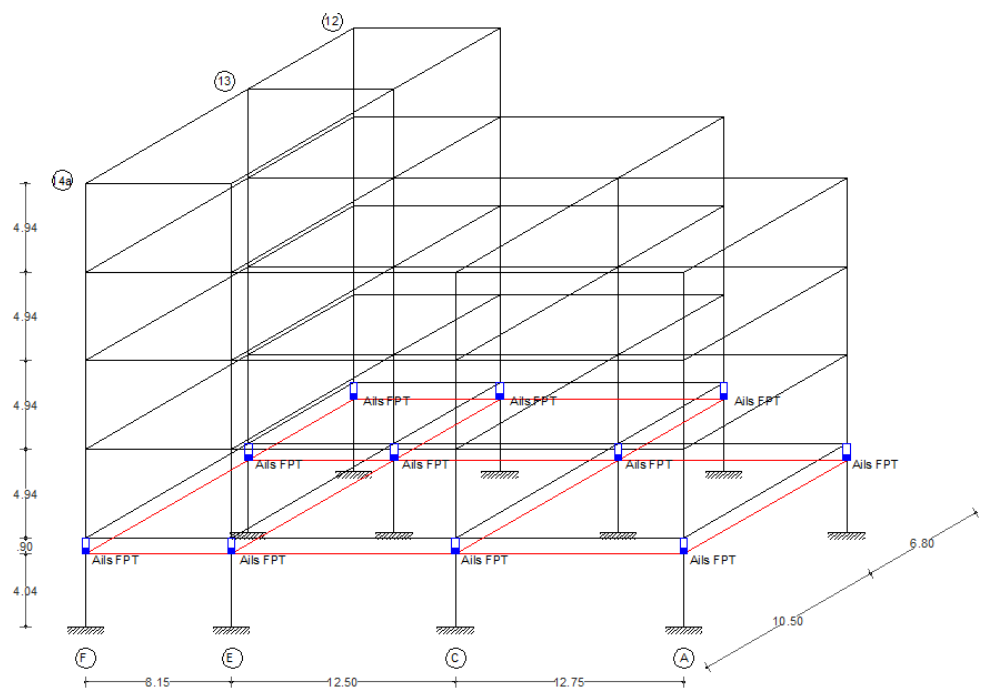

Figura 17 Vista tridimensional bloque 3

\subsubsection{Información del edificio}

Se inicia el ingreso de datos al llenar la primera matriz correspondiente a la información del edificio.

Tabla 6 Matriz de información del edificio

\begin{tabular}{|c|c|c|c|c|c|}
\hline Pisos & $\begin{array}{c}\text { Altura de } \\
\text { piso (m) }\end{array}$ & Area (m2) & $\begin{array}{c}\text { Factor de } \\
\text { altura }\end{array}$ & $\begin{array}{c}\text { Factor por } \\
\text { materiales } \\
\text { peligrosos }\end{array}$ & $\begin{array}{c}\text { Factor por } \\
\text { ocupación }\end{array}$ \\
\hline Piso 1 & 4,94 & 491,20 & 1,08 & 1 & 1,8 \\
\hline Piso 2 & 4,94 & 491,20 & 1,08 & 1 & 1,8 \\
\hline Piso 3 & 4,94 & 491,20 & 1,08 & 1 & 1,8 \\
\hline Piso 4 & 4,94 & 491,20 & 1,08 & 1 & 1,8 \\
\hline Piso 5 & 4,94 & 141,00 & 1,08 & 1 & 1,8 \\
\hline Cubierta & 0,00 & 141,00 & 1,08 & 1 & 1,8 \\
\hline
\end{tabular}

- Para el factor por altura se toma un valor de 1.08 por tener 5 pisos, valor propuesto según FEMA P-58, Volumen 1.

- Para el factor por materiales peligrosos se toma un valor de 1.00 por ser un edificio moderno con una cantidad de materiales peligrosos insignificante.

- Para el factor por ocupación se toma un valor de 1.80 por ser un Centro de Investigaciones ocupado, valor propuesto según FEMA P-58, Volumen 1.

Se utilizó un trabajador por cada $92.90 \mathrm{~m}^{2}$, recomendado en el FEMA P58 , Volumen 1. 
Para el umbral de pérdida se utiliza un valor predeterminado de 1 que maximiza la cantidad de información de los resultados que se obtendrán en una evaluación.

Para conocer el costo total de construcción del bloque 3 se tiene como dato inicial el costo total de construcción de todos los bloques del nuevo Centro de Investigaciones Científicas y de Post Grado de la Universidad de Fuerzas Armadas ESPE, para lo cual el costo total del proyecto se divide para el área total de construcción y se asume un valor de 1000 USD / M2, dicho valor se multiplica por el área total del bloque 3. El tiempo de construcción se asume 180 días.

Tabla 7 Desglose de costos para el Bloque 3 con y sin aisladores

\begin{tabular}{|c|c|r|}
\hline Costo total del proyecto & USD & $\$ 20.422 .481,73$ \\
\hline Total proyecto & M2 & 22504,00 \\
\hline Costo calculado por m2 & USD & $\$ 907,50$ \\
\hline Costo estimado por m2 & USD & $\$ 1.000,00$ \\
\hline 4 pisos & M2 & 491,20 \\
\hline 1 piso & M2 & 141,00 \\
\hline Total bloque 3 & M2 & 2105,80 \\
\hline $\begin{array}{c}\text { Costo sin aisladores } \\
\text { bloque 3 }\end{array}$ & USD & $\$ 1.952 .970,29$ \\
\hline $\begin{array}{c}\text { Costo con aisladores } \\
\text { bloque 3 }\end{array}$ & USD & $\$ 2.105 .800,00$ \\
\hline $\begin{array}{c}\text { Tiempo de construcción } \\
\text { DIAS }\end{array}$ & 180,00 \\
\hline
\end{tabular}

\subsubsection{Población del Bloque 3}

Para la población del Bloque 3 se selecciona en PACT Centro de Investigaciones, el cual consta de porcentajes de ocupación por cada hora, predeterminados para el tipo de ocupación seleccionado.

\subsubsection{Elementos estructurales y no estructurales Bloque 3}

Los elementos que se consideran como estructurales son: columnas, vigas y losa; y los elementos no estructurales son: paredes de mampostería, paredes interiores, pisos, techos, aire, tuberías de agua potable.

Los elementos estructurales y no estructurales que se seleccionan en PACT, se describen a continuación, y cada uno de estos elementos cuenta con su respectiva curva de fragilidad. 
Tabla 8 Elementos de acero

\begin{tabular}{|c|c|}
\hline Código en PACT & \multicolumn{1}{|c|}{ Descripción } \\
\hline B 1035.032 & $\begin{array}{c}\text { Viga unida a dos lados de la } \\
\text { columna, resistente a la flexión, } \\
\text { con una profundidad } \geq \text { W30 }\end{array}$ \\
\hline B 1035.022 & $\begin{array}{l}\text { Viga unida a un solo lado de } \\
\text { la columna, resistente a la flexión, } \\
\text { con una profundidad } \geq \text { W30 }\end{array}$ \\
\hline
\end{tabular}

Tabla 9 Elementos de hormigón armado

\begin{tabular}{|c|c|}
\hline Código en PACT & Descripción \\
\hline B $1041.003 \mathrm{a}$ & $\begin{array}{l}\text { ACl 3-18 SMF Columna y } \\
\text { viga de } 91.44 \mathrm{~cm} \times 91.44 \mathrm{~cm} \text { con } \\
\text { viga a un solo lado }\end{array}$ \\
\hline B 1041.003 b & $\begin{array}{l}\mathrm{ACl} 3-18 \text { SMF Columna y } \\
\text { viga de } 91.44 \mathrm{~cm} \times 91.44 \mathrm{~cm} \text { con } \\
\text { viga a ambos lados }\end{array}$ \\
\hline B 1049.011 & 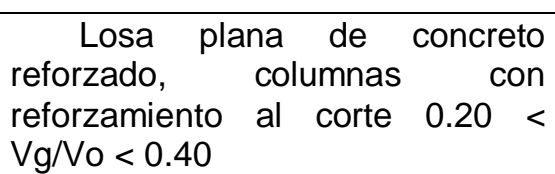 \\
\hline
\end{tabular}

Tabla 10 Elementos no estructurales

\begin{tabular}{|c|l|}
\hline Código en PACT & \multicolumn{1}{|c|}{ Descripción } \\
\hline B 1051.012 & $\begin{array}{l}\text { Pared de mampostería con } \\
\text { reforzamiento ordinario, con } \\
\text { elementos } \\
\text { parcialmente } \\
\text { espesor, mayor que } 3.65 \mathrm{~m} \mathrm{de} \\
\text { altura }\end{array}$ \\
\hline C 1011.001 b & $\begin{array}{l}\text { Paredes para particiones, tipo } \\
\text { gypsum con tiras de metal, altura } \\
\text { parcial, fijado abajo con } \\
\text { arriostramientos laterales arriba }\end{array}$ \\
\hline C 3027.001 & $\begin{array}{l}\text { Suelo técnico sísmicamente } \\
\text { no calificado }\end{array}$ \\
\hline C 3032.001 a & $\begin{array}{l}\text { Techo suspendido, SDC A, B, } \\
\text { Area (A): A 23.22 m2 , solo } \\
\text { soporte vertical }\end{array}$ \\
\hline
\end{tabular}




\begin{tabular}{|c|c|}
\hline D 2021.011 a & $\begin{array}{c}\text { Tuberías de agua fría } \\
\text { (diámetro }>6.35 \mathrm{~cm} \text { ), clasificación } \\
\text { SDC A o B, fragilidad de tuberías }\end{array}$ \\
\hline D 3041.041 a & $\begin{array}{c}\text { Sistema de aire acondicionado } \\
\text { variable (VAV) caja de bobina en } \\
\text { línea, SDC A o B }\end{array}$ \\
\hline
\end{tabular}

\subsubsection{Fragilidad de colapso}

En este análisis no se incluye la fragilidad de colapso, ya que no se cuenta con datos de tasas medias de fatalidad y lesiones de eventos ocurridos en el pasado. Para la fragilidad de colapso únicamente se cuenta con la aceleración media de colapso, la misma que se obtuvo del análisis estático no lineal (PUSHOVER) y se describe posteriormente.

\subsubsection{Resultados del análisis estructural}

Los resultados de análisis estructural se obtienen del análisis modal espectral, para la evaluación del Bloque 3 los parámetros de demanda son derivas y aceleraciones de piso.

(Aguiar R. , 2012). El fundamento del método modal espectral, es el desacoplamiento de la ecuación diferencial que gobierna los problemas dinámicos, que se tiene a continuación.

$$
M \ddot{q}+C \dot{q}+K q=Q
$$

Donde $\mathrm{M}$ es la matriz de masas, $\mathrm{C}$ la matriz de amortiguamiento y $\mathrm{K}$ la matriz de rigidez, como resultado del desacoplamiento se tienen las ecuaciones de desplazamientos y fuerzas modales para el cálculo de una determinada estructura.

$$
\begin{aligned}
q^{i} & =\gamma\left(\frac{T}{2 \pi}\right)^{2} A d \phi \\
Q^{i} & =\gamma A d M \phi
\end{aligned}
$$

Se presentan los resultados de análisis modal espectral del bloque 3, modelado sin aisladores.

Tabla 11 Resultado del análisis modal espectral (Aguiar R. , 2012)

\begin{tabular}{|l|c|c|}
\hline $\begin{array}{c}\text { Período de } \\
\text { Recurrencia } \\
\text { (años) }\end{array}$ & $\begin{array}{c}\mathbf{4 7 5} \\
\text { (DBE) }\end{array}$ & $\begin{array}{c}\mathbf{9 0 0} \\
\text { (MCE) }\end{array}$ \\
\hline Piso5-Cubierta & 0,0055 & 0,0077 \\
\hline Piso 4-5 & 0,0105 & 0,0147 \\
\hline Piso 3-4 & 0,0150 & 0,0212 \\
\hline Piso 2-3 & 0,0160 & 0,0225 \\
\hline Piso 1-2 & 0,0075 & 0,0106 \\
\hline
\end{tabular}


Al tener los resultados de las derivas con el sismo de diseño DBE (475 años) y sismo máximo creíble MCE (900 años), se requiere adicionalmente obtener derivas en un rango entre 475 años a 2400 años, que exige la metodología FEMA P-58 para el ingreso de datos en el programa PACT.

Se toma un valor intermedio entre las derivas de DBE y MCE, se obtiene una deriva para un período de recurrencia de 600 años. Se calcula la media de las derivas de 600 y 900 años, para luego deducir un factor; se divide la media de 900 años para la media de 600 años, el mismo que permite obtener derivas cada 300 años hasta llegar a 2400 años.

$$
\begin{gathered}
\Delta_{600}=\frac{\Delta_{475}+\Delta_{900}}{2} \\
\text { Factor }=\frac{\Delta_{\text {media } 900}}{\Delta_{\text {media } 600}} \\
\text { Factor }=\frac{0.0153}{0.0131}=1.169
\end{gathered}
$$

El programa PACT también requiere un valor de dispersión por cada grupo de datos ingresados.

$$
\text { Dispersión }=\sqrt{\frac{\left(\sum_{i}^{n}(\Delta i-\Delta m e d)^{2}\right)}{n}}
$$

Los resultados del análisis previo se presentan en la siguiente tabla:

Tabla 12 Derivas calculadas para el rango entre 475 y 2400 años sin aisladores (Aguiar R. , 2012)

\begin{tabular}{|l|c|c|c|c|c|c|c|c|c|}
\hline Media & 0,011 & 0,013 & 0,015 & 0,018 & 0,021 & 0,025 & 0,029 & 0,031 & 0,033 \\
\hline Dispersión & 0,004 & 0,005 & 0,006 & 0,007 & 0,008 & 0,009 & 0,011 & 0,012 & 0,013 \\
\hline \multicolumn{7}{|c|}{ DERIVAS RESULTADO DEL ANÁLISIS MODAL ESPECTRAL } \\
\begin{tabular}{|l|l|l|l|l|l|l|l|} 
Período de \\
Recurrencia (años)
\end{tabular} & $\mathbf{4 7 5}$ (DBE) & $\mathbf{6 0 0}$ & $\mathbf{9 0 0}$ (MCE) & $\mathbf{1 2 0 0}$ & $\mathbf{1 5 0 0}$ & $\mathbf{1 8 0 0}$ & $\mathbf{2 1 0 0}$ & $\mathbf{2 2 5 0}$ & $\mathbf{2 4 0 0}$ \\
\hline Piso5-Cubierta & 0,006 & 0,007 & 0,008 & 0,009 & 0,010 & 0,012 & 0,014 & 0,016 & 0,017 \\
\hline Piso 4-5 & 0,011 & 0,013 & 0,015 & 0,017 & 0,020 & 0,023 & 0,027 & 0,030 & 0,032 \\
\hline Piso 3-4 & 0,015 & 0,018 & 0,021 & 0,025 & 0,029 & 0,034 & 0,040 & 0,043 & 0,046 \\
\hline Piso 2-3 & 0,016 & 0,019 & 0,023 & 0,026 & 0,031 & 0,036 & 0,042 & 0,046 & 0,049 \\
\hline Piso 1-2 & 0,008 & 0,009 & 0,011 & 0,012 & 0,014 & 0,017 & 0,020 & 0,021 & 0,023 \\
\hline
\end{tabular}


Para el cálculo de las aceleraciones de piso se utiliza el mismo procedimiento descrito anteriormente en la obtención de las derivas.

Tabla 13 Aceleraciones de piso calculadas para el rango entre 475 y 2400 años sin aisladores (Aguiar R. , 2012)

\begin{tabular}{|c|c|c|c|c|c|c|c|c|c|}
\hline Media & 0,278 & 0,380 & 0,481 & 0,610 & 0,774 & 0,982 & 1,245 & 1,412 & 1,579 \\
\hline Dispersión & 0,048 & 0,034 & 0,031 & 0,039 & 0,049 & 0,062 & 0,079 & 0,090 & 0,100 \\
\hline \multicolumn{10}{|c|}{ ACELERACIONES DE PISO RESULTADO DEL ANÁLISIS MODAL ESPECTRAL } \\
\hline $\begin{array}{l}\text { Período de } \\
\text { Recurrencia (años) }\end{array}$ & 475 (DBE) & 600 & 900 (MCE) & 1200 & 1500 & 1800 & 2100 & 2250 & 2400 \\
\hline Cubierta & 0,533 & 0,546 & 0,558 & 0,708 & 0,898 & 1,138 & 1,444 & 1,637 & 1,831 \\
\hline Piso 5 & 0,485 & 0,496 & 0,508 & 0,644 & 0,816 & 1,035 & 1,312 & 1,488 & 1,664 \\
\hline Piso 4 & 0,262 & 0,406 & 0,550 & 0,697 & 0,884 & 1,121 & 1,422 & 1,613 & 1,803 \\
\hline Piso 3 & 0,232 & 0,359 & 0,486 & 0,617 & 0,782 & 0,992 & 1,258 & 1,426 & 1,595 \\
\hline Piso 2 & 0,244 & 0,378 & 0,512 & 0,650 & 0,824 & 1,045 & 1,325 & 1,502 & 1,680 \\
\hline Piso 1 & 0,167 & 0,259 & 0,351 & 0,445 & 0,565 & 0,716 & 0,908 & 1,030 & 1,152 \\
\hline
\end{tabular}

$$
\text { Factor }=\frac{0.4814}{0.3796}=1.268
$$

A continuación se presentan los resultados del análisis modal espectral del bloque 3 , modelado con aisladores de base triple péndulo de fricción en todos los ejes de columnas.

Tabla 14 Resultado del análisis modal espectral (Aguiar R. , 2012)

\begin{tabular}{|l|c|c|}
\hline $\begin{array}{c}\text { Período de } \\
\text { Recurrencia } \\
\text { (años) }\end{array}$ & $\begin{array}{c}\mathbf{4 7 5} \\
\text { (DBE) }\end{array}$ & $\begin{array}{c}\mathbf{9 0 0} \\
\text { (MCE) }\end{array}$ \\
\hline Piso5-Cubierta & 0,169 & 0,230 \\
\hline Piso 4-5 & 0,138 & 0,191 \\
\hline Piso 3-4 & 0,125 & 0,174 \\
\hline Piso 2-3 & 0,123 & 0,171 \\
\hline Piso 1-2 & 0,131 & 0,181 \\
\hline
\end{tabular}


Tabla 15 Derivas calculadas para el rango entre 475 y 2400 años con aisladores (Aguiar R. , 2012)

\begin{tabular}{|c|c|c|c|c|c|c|c|c|c|}
\hline Media & 0,002 & 0,003 & 0,003 & 0,003 & 0,004 & 0,004 & 0,005 & 0,005 & 0,006 \\
\hline Dispersión & 0,001 & 0,001 & 0,001 & 0,001 & 0,002 & 0,002 & 0,002 & 0,002 & 0,003 \\
\hline \multicolumn{10}{|c|}{ DERIVAS RESULTADO DEL ANÁLISIS MODAL ESPECTRAL } \\
\hline $\begin{array}{c}\text { Período de } \\
\text { Recurrencia (años) }\end{array}$ & 475 (DBE) & 600 & 900 (MCE) & 1200 & 1500 & 1800 & 2100 & 2250 & 2400 \\
\hline Piso5-Cubierta & 0,002 & 0,002 & 0,002 & 0,002 & 0,003 & 0,003 & 0,004 & 0,004 & 0,004 \\
\hline Piso 4-5 & 0,002 & 0,002 & 0,003 & 0,003 & 0,003 & 0,004 & 0,005 & 0,005 & 0,005 \\
\hline Piso 3-4 & 0,003 & 0,003 & 0,004 & 0,005 & 0,005 & 0,006 & 0,007 & 0,008 & 0,008 \\
\hline Piso 2-3 & 0,003 & 0,004 & 0,005 & 0,005 & 0,006 & 0,007 & 0,008 & 0,009 & 0,009 \\
\hline Piso 1-2 & 0,001 & 0,001 & 0,001 & 0,001 & 0,002 & 0,002 & 0,002 & 0,002 & 0,002 \\
\hline
\end{tabular}

$$
\text { Factor }=\frac{0.0029}{0.0025}=1.151
$$

Tabla 16 Aceleraciones de piso calculadas para el rango entre 475 y 2400 años con aisladores (Aguiar R. , 2012)

\begin{tabular}{|c|c|c|c|c|c|c|c|c|c|}
\hline \\
\hline Media & 0,137 & 0,163 & 0,189 & $3,1 \mathrm{E}-02$ & $5,0 \mathrm{E}-03$ & $8,2 \mathrm{E}-04$ & $1,3 \mathrm{E}-04$ & $7,8 \mathrm{E}-05$ & $2,2 \mathrm{E}-05$ \\
\hline Dispersión & $7,4 \mathrm{E}-03$ & $8,5 \mathrm{E}-03$ & $9,6 \mathrm{E}-03$ & $1,6 \mathrm{E}-03$ & $2,6 \mathrm{E}-04$ & $4,2 \mathrm{E}-05$ & $6,8 \mathrm{E}-06$ & $4,0 \mathrm{E}-06$ & $1,1 \mathrm{E}-06$ \\
\hline \multicolumn{10}{|c|}{ ACELERACIONES DE PISO RESULTADO DEL ANÁLISIS MODAL ESPECTRAL } \\
\hline $\begin{array}{c}\text { Período de } \\
\text { Recurrencia (años) }\end{array}$ & 475 (DBE) & 600 & 900 (MCE) & 1200 & 1500 & 1800 & 2100 & 2250 & 2400 \\
\hline Piso5-Cubierta & 0,169 & 0,199 & 0,230 & 0,038 & $6,1 \mathrm{E}-03$ & $1,0 \mathrm{E}-03$ & $1,6 \mathrm{E}-04$ & 9,5E-05 & $2,7 \mathrm{E}-05$ \\
\hline Piso 4-5 & 0,138 & 0,165 & 0,191 & 0,031 & $5,1 \mathrm{E}-03$ & 8,3E-04 & $1,4 \mathrm{E}-04$ & 7,9E-05 & $2,2 \mathrm{E}-05$ \\
\hline Piso 3-4 & 0,125 & 0,149 & 0,174 & 0,028 & 4,6E-03 & $7,6 \mathrm{E}-04$ & $1,2 \mathrm{E}-04$ & 7,2E-05 & $2,0 \mathrm{E}-05$ \\
\hline Piso 2-3 & 0,123 & 0,147 & 0,171 & 0,028 & $4,6 \mathrm{E}-03$ & $7,5 \mathrm{E}-04$ & $1,2 \mathrm{E}-04$ & $7,1 \mathrm{E}-05$ & $2,0 \mathrm{E}-05$ \\
\hline Piso 1-2 & 0,131 & 0,156 & 0,181 & 0,029 & $4,8 \mathrm{E}-03$ & $7,9 \mathrm{E}-04$ & $1,3 \mathrm{E}-04$ & $7,5 \mathrm{E}-05$ & $2,1 \mathrm{E}-05$ \\
\hline
\end{tabular}

$$
\text { Factor }=\frac{0.1893}{0.1633}=1.159
$$

\subsubsection{Deriva residual}

Se puede ingresar un valor medio de deriva residual con su dispersión, con esto se genera una curva de fragilidad para un estado de daño irreparable. PACT utiliza un valor medio de deriva residual de 0.015 con una dispersión de 0.30. La opción de deriva residual se puede omitir para el análisis. 


\subsubsection{Curva de peligrosidad sísmica}

La curva de peligrosidad describe las características de sismicidad del sitio, en función del tipo de suelo y el factor de zona Z. Las curvas de peligro sísmico se grafican con aceleraciones de suelo y tasa anual de excedencia, y tienen curvas para diferentes períodos fundamentales de las estructuras.

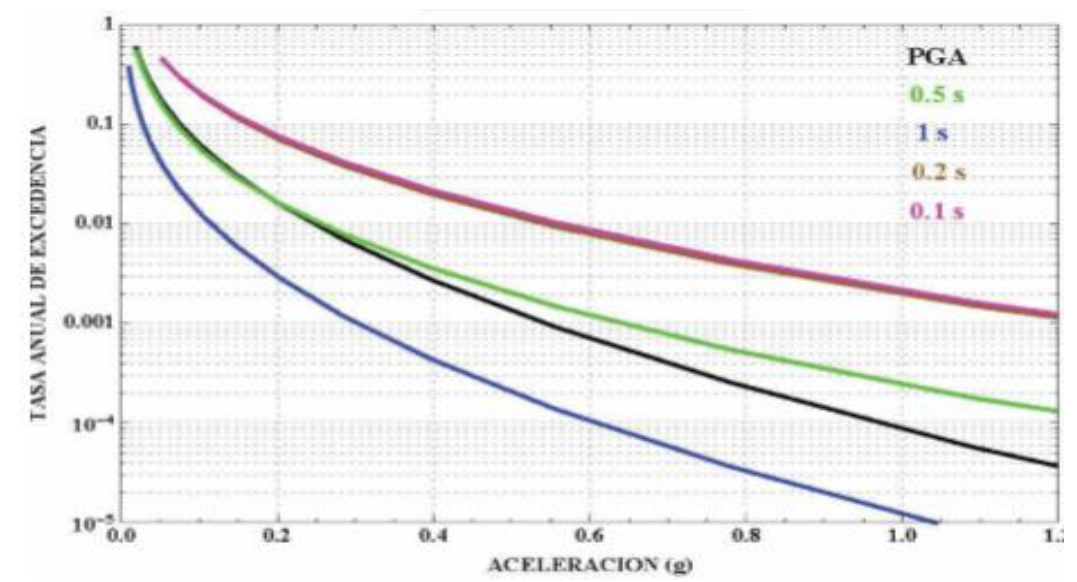

Figura 18 Curva peligro sísmico Quito (NEC, 2014)

Para generar la curva de peligro sísmico del Bloque 3 se aplicaron las fórmulas y la metodología propuesta en el FEMA P-58. Se tiene un período fundamental para la estructura de $1.19 \mathrm{~s}$ sin aisladores y $3.60 \mathrm{~s}$ con aisladores, para el primer caso se trabaja con la curva de peligrosidad del NEC 14 para un período de un segundo por ser la más aproximada (véase figura 18).

\section{Estructura SIN AISLADORES}

(FEMA P-58 VOL 1, 2012). Se calcula la aceleración espectral efectiva según el ASCE/SEI 7, al aplicar la siguiente fórmula:

$$
S_{a D}=\frac{V}{W} * R
$$

(Aguiar R Mora D, 2015). Siendo: V el cortante basal de colapso calculado en CEINCI - LAB, W el peso total de la estructura, y $\mathrm{R}$ el factor de reducción de fuerzas sísmicas.

Tabla 17 Aceleración espectral efectiva sin aisladores

\begin{tabular}{|c|c|c|}
\hline Parámetro & Valor & Unidad \\
\hline V & 270,00 & $\mathrm{~T}$ \\
\hline W & 2059,12 & $\mathrm{~T}$ \\
\hline R & 3 & Adimensional \\
\hline SaD & 0,39 & $\mathrm{~g}$ \\
\hline
\end{tabular}


(FEMA P-58 VOL 1, 2012). A continuación se calcula la capacidad de colapso media, en función del período fundamental del edificio $\mathrm{Sa}(\mathrm{T})$, se utiliza la fórmula que corresponde a períodos estructurales $\geq 0.60 \mathrm{~s}$

$$
\overline{S_{a}}(T)=4 * S_{a D}=4 * 0.39=1.57
$$

Convertir la capacidad media calculada en el período fundamental del edificio al período medio fundamental del edificio de $\mathrm{T}$ a $\overline{\mathrm{T}}$.

$$
\begin{gathered}
\overline{\mathrm{T}}=\frac{\mathrm{Txx}+\mathrm{Tyy}}{2}=\frac{1.19+1.19}{2}=1.19 \\
\widehat{S a}(\overline{\mathrm{T}})=\frac{T}{\overline{\mathrm{T}}} * \overline{S_{a}}(T) \\
\widehat{S a}(\overline{\mathrm{T}})=\frac{1.19}{1.19} * 1.57=1.57(g)
\end{gathered}
$$

Para encontrar los valores de la curva de peligrosidad, se necesita una aceleración mínima y una aceleración máxima dentro de las que se desarrolla la curva de peligro sísmico, para el ingreso en el programa. Se utiliza las siguientes fórmulas:

$$
\begin{gathered}
\text { Sa min }=\frac{0.05}{T} \text { para } \mathrm{T}>1.0 \mathrm{~s} \\
\text { Sa } \min =\frac{0.05}{1.19}=0.042 \mathrm{~g}
\end{gathered}
$$

Para la aceleración máxima se debe tomar el menor valor entre: Sa max en función de una tasa anual de excedencia para 2500 años, $\Lambda=1 / 2500=0.0004$ y el valor obtenido con la fórmula (12).

$$
\begin{gathered}
\text { Sa } \max (\Lambda=0.0004)=0.41 \mathrm{~g} \\
\text { Sa } \max =2 * \widehat{S a}(\overline{\mathrm{T}})=2 *(1.57)=3.15 \mathrm{~g}
\end{gathered}
$$

Se toma Sa $\max =\mathbf{0 . 4 1} \mathbf{g}$ para la curva de peligrosidad sísmica. intervalo.

Definidos los valores de Sa mín y Sa máx, se encuentra el valor del

$$
\text { Intervalo }=\frac{\text { Sa max }- \text { Sa min }}{8}=\frac{0.41-0.042}{8}=0.04
$$

Tabla 18 Aceleraciones y tasa anual de excedencia sin aisladores

\begin{tabular}{|c|c|}
\hline $\mathbf{S a}(\mathbf{g})$ & $\boldsymbol{\Lambda}$ \\
\hline 0,042 & 0,08 \\
\hline 0,065 & 0,035 \\
\hline 0,111 & 0,011 \\
\hline 0,157 & 0,0055 \\
\hline 0,203 & 0,003 \\
\hline
\end{tabular}




\begin{tabular}{|c|c|}
0,249 & 0,0018 \\
\hline 0,295 & 0,001 \\
\hline 0,341 & 0,0007 \\
\hline 0,387 & 0,00045 \\
\hline 0,410 & 0,0004 \\
\hline
\end{tabular}

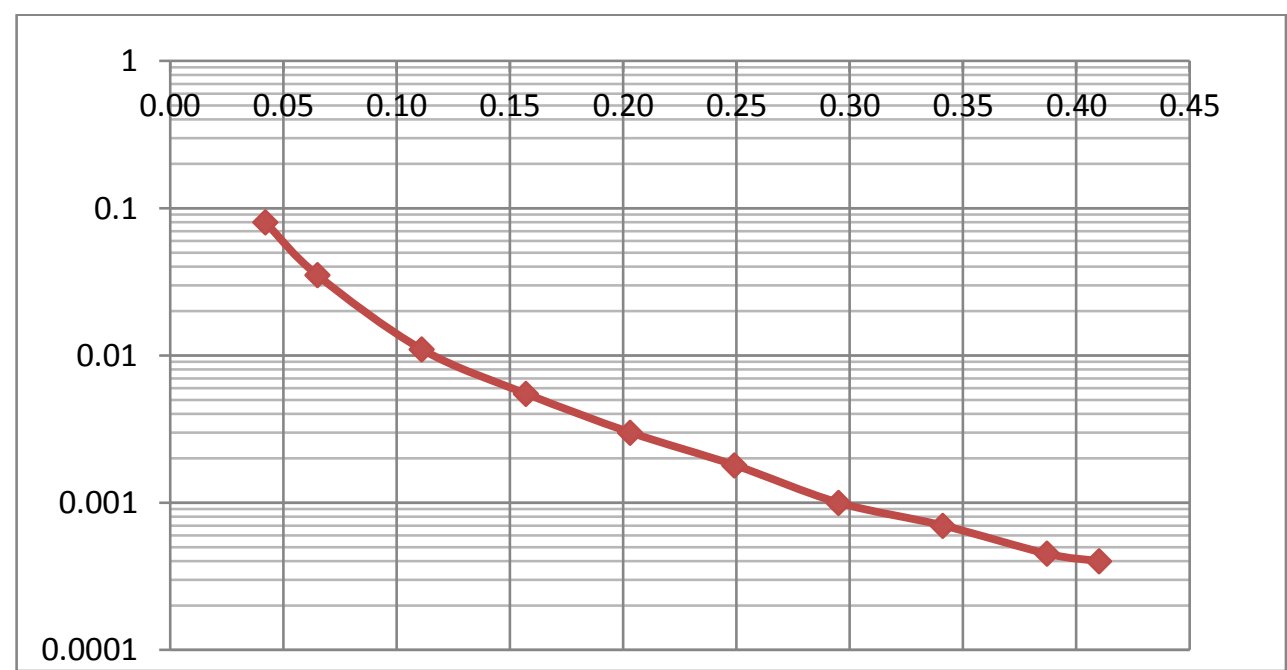

Figura 19 Curva peligrosidad sísmica Bloque 3 sin aisladores

\section{Estructura CON AISLADORES TRIPLE PÉNDULO DE FRICCIÓN (FPT)}

Se realiza el mismo procedimiento descrito anteriormente, con el valor de cortante basal de colapso de la estructura modelada con aisladores.

Tabla 19 Aceleración espectral efectiva con aisladores

\begin{tabular}{|c|c|c|}
\hline Parámetro & Valor & Unidad \\
\hline V & 180,00 & $\mathrm{~T}$ \\
\hline $\mathrm{W}$ & 2059,12 & $\mathrm{~T}$ \\
\hline $\mathrm{R}$ & 1,4 & Adimensional \\
\hline $\mathrm{SaD}$ & 0,12 & $\mathrm{~g}$ \\
\hline
\end{tabular}

Se calcula la capacidad de colapso media, en función del período fundamental del edificio Sa (T).

\begin{tabular}{|l|l|l|}
\hline SaD & 0,12 & $\mathrm{~g}$ \\
\hline Samed $(\mathrm{T})$ & 0,49 & $\mathrm{~g}$ \\
\hline
\end{tabular}

Se convierte la capacidad media calculada en el período fundamental del edificio al período medio fundamental del edificio de $\mathrm{T}$ a $\overline{\mathrm{T}}$. 


$$
\begin{gathered}
\overline{\mathrm{T}}=\frac{3.60+3.60}{2}=3.60 \\
\widehat{S a}(\overline{\mathrm{T}})=\frac{3.60}{3.60} * 0.49=0.49(\mathrm{~g})
\end{gathered}
$$

Aceleración mínima y máxima

$$
\begin{gathered}
\mathrm{T}=3.6 \mathrm{~s} \rightarrow \text { Sa min }=0.05 \mathrm{~g} \\
\text { Sa } \max =2 *(0.49)=0.98 \mathrm{~g}
\end{gathered}
$$

Para la aceleración máxima, Sa max en función de una tasa anual de excedencia para 2500 años $(\Lambda=0.0004)$, se debe generar una curva de peligrosidad para Quito con un período fundamental de $3.60 \mathrm{~s}$, ya que no existe en el NEC 14.

Se obtiene los valores de aceleraciones máximas y mínimas con las tasas anuales de excedencia $\Lambda=0.0021$ y $\Lambda=0.0010$ (475 y 1000 años) de la curva de peligrosidad de $1 \mathrm{~s}$.

Tabla 20 Aceleraciones máx y mín

\begin{tabular}{|c|c|c|c|}
\hline Tr & 475 y 1000 & años & $\lambda$ \\
\hline Samin T=1s & 0,24 & $\mathrm{~g}$ & 0,0021 \\
Samax T=1s & 0.30 & $\mathrm{~g}$ & 0.0010 \\
\hline
\end{tabular}

Se calcula la aceleración para sismo máximo creíble (MCE), al multiplicar la aceleración con el sismo de diseño (DBE) por 1.4

Tabla 21 Aceleraciones espectrales

\begin{tabular}{|c|l|l|}
\hline T & 3,62 & $\mathrm{~s}$ \\
\hline Sa (DBE) & 0,17 & $\mathrm{~g}$ \\
Sa (MCE) & 0.24 & $\mathrm{~g}$ \\
\hline
\end{tabular}

Los factores se obtienen:

$$
\text { Factor } 1=\frac{\mathrm{Sa}(\mathrm{DBE})}{\mathrm{Sa} \min }
$$

Factor $1=\frac{0.17}{0.24}=0.708$

$$
\text { Factor } 2=\frac{\mathrm{Sa}(\mathrm{MCE})}{\mathrm{Sa} \max }
$$

Factor $2=\frac{0.24}{0.30}=0.793$ 
Entre los dos factores se calcula un promedio

$$
\begin{gathered}
\text { Factor promedio }=\frac{\text { Factor } 1+\text { Factor } 2}{2} \\
\text { Factor promedio }=\frac{0.708+0.793}{2}=0.75
\end{gathered}
$$

Finalmente para generar la curva de peligrosidad sísmica para $3.60 \mathrm{~s}$, se multiplica el Factor promedio por las aceleraciones de $1 \mathrm{~s}$.

Tabla 22 Aceleraciones y tasa anual de excedencia para diferentes

\begin{tabular}{|c|c|c|c|}
\hline \multicolumn{2}{|c|}{ Para $\mathrm{T}=1 \mathrm{~s}$} & \multicolumn{2}{|c|}{ Para $\mathrm{T}=3,6 \mathrm{~s}$} \\
\hline Sa (g) & $\lambda$ & Sa (g) & $\lambda$ \\
\hline 0,01 & 0,290000 & 0,0075 & 0,2900000 \\
\hline 0,05 & 0,040000 & 0,0375 & 0,0400000 \\
\hline 0,10 & 0,010000 & 0,0751 & 0,0100000 \\
\hline 0,15 & 0,005000 & 0,1126 & 0,0050000 \\
\hline 0,20 & 0,003000 & 0,1502 & 0,0030000 \\
\hline 0,25 & 0,001800 & 0,1877 & 0,0018000 \\
\hline 0,30 & 0,001000 & 0,2253 & 0,0010000 \\
\hline 0,35 & 0,000600 & 0,2628 & 0,0006000 \\
\hline 0,40 & 0,000300 & 0,3003 & 0,0003000 \\
\hline 0,45 & 0,000280 & 0,3379 & 0,0002800 \\
\hline 0,50 & 0,000200 & 0,3754 & 0,0002000 \\
\hline 0,55 & 0,000140 & 0,4130 & 0,0001400 \\
\hline 0,60 & 0,000100 & 0,4505 & 0,0001000 \\
\hline 0,65 & 0,000070 & 0,4880 & 0,0000700 \\
\hline 0,70 & 0,000060 & 0,5256 & 0,0000600 \\
\hline 0,75 & 0,000040 & 0,5631 & 0,0000400 \\
\hline 0,80 & 0,000030 & 0,6007 & 0,0000300 \\
\hline 0,85 & 0,000025 & 0,6382 & 0,0000250 \\
\hline 0,90 & 0,000020 & 0,6758 & 0,0000200 \\
\hline 0,95 & 0,000015 & 0,7133 & 0,0000150 \\
\hline 1,00 & 0,000012 & 0,7508 & 0,0000120 \\
\hline 1,05 & 0,000010 & 0,7884 & 0,0000100 \\
\hline 1,10 & 0,000008 & 0,8259 & 0,0000080 \\
\hline 1,15 & 0,000006 & 0,8635 & 0,0000060 \\
\hline 1,20 & 0,000004 & 0,9010 & 0,0000040 \\
\hline
\end{tabular}
períodos 


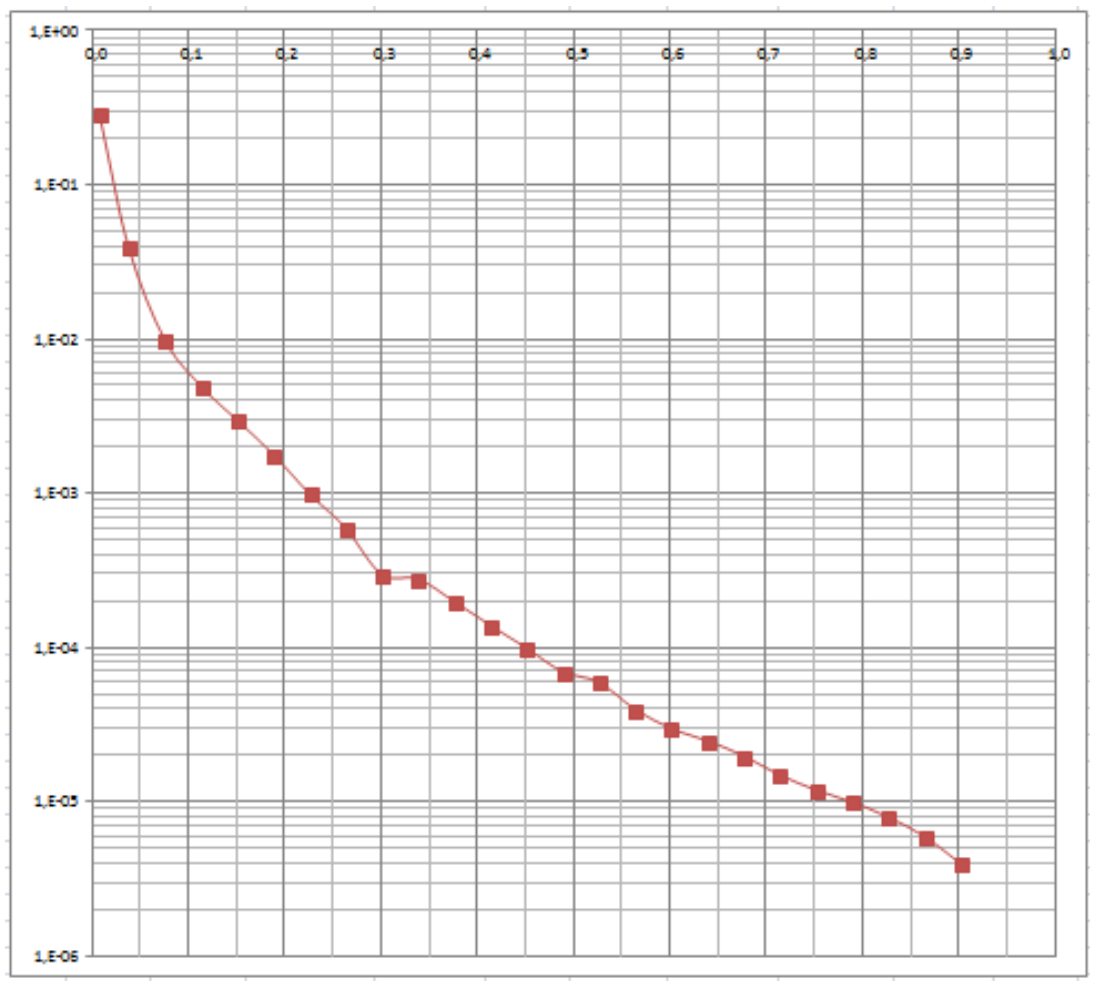

Figura 20 Curva peligrosidad sísmica para Quito con un período fundamental de $3.60 \mathrm{~s}$

Se ingresa a la curva de peligrosidad que se presenta en la figura 20 con $\Lambda=0.0004$ (2400 años) para obtener Sa máx

Sa $\max (\Lambda=0.0004)=0.28 \mathrm{~g}$

Se toma Sa máx $=0.28 \mathrm{~g}$ para la curva de peligrosidad sísmica.

Tabla 23 Aceleraciones y tasa anual de excedencia con aisladores

\begin{tabular}{|c|c|}
\hline Sa (g) & $\boldsymbol{\lambda}$ \\
\hline 0,050 & 0,030000 \\
\hline 0,064 & 0,015 \\
\hline 0,093 & 0,008 \\
\hline 0,122 & 0,005 \\
\hline 0,151 & 0,003 \\
\hline 0,179 & 0,002 \\
\hline 0,208 & 0,0012 \\
\hline 0,237 & 0,0009 \\
\hline 0,266 & 0,00062 \\
\hline 0,280 & 0,000400 \\
\hline
\end{tabular}




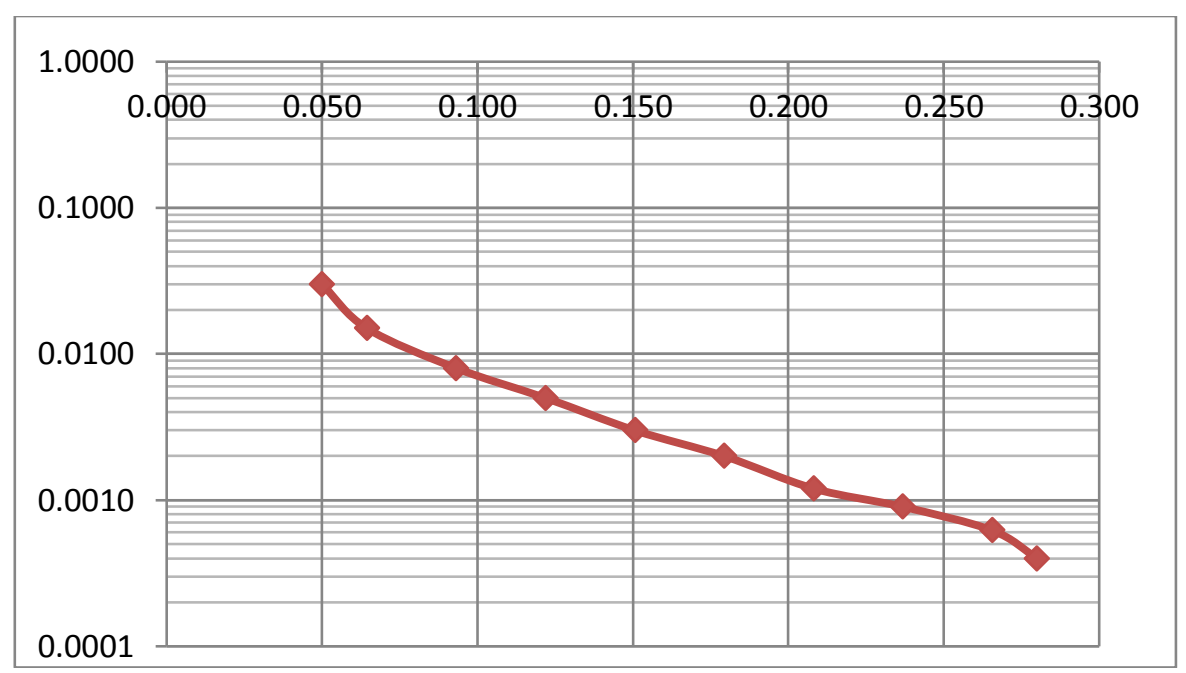

Figura 21 Curva peligrosidad sísmica Bloque 3 con aisladores

\section{RESULTADOS Y DISCUCIÓN BLOQUE 3}

\subsubsection{Costos de reparación y calificación según $\mathrm{REDI}^{\mathrm{TM}}$}

Se presenta los costos de construcción, costos de reparación y porcentajes de pérdida financiera que se obtienen de la evaluación en el programa PACT, para el Bloque 3 modelado con y sin aisladores.

Tabla 24 Resultados de la evaluación en PACT

\begin{tabular}{|c|c|c|c|}
\hline Edificación / Costos & $\begin{array}{c}\text { Costo Total de } \\
\text { Construcción }\end{array}$ & $\begin{array}{c}\text { Costo de } \\
\text { Reparación }\end{array}$ & \% Pérdida Financiera \\
\hline $\begin{array}{c}\text { Bloque 3 Sin } \\
\text { Aisladores }\end{array}$ & $\$ 1.952 .970,00$ & $\$ 165.509,55$ & $8,47 \%$ \\
\hline $\begin{array}{c}\text { Bloque 3 Con } \\
\text { Aisladores }\end{array}$ & $\$ 2.105 .800,00$ & $\$ 68.115,60$ & $3,23 \%$ \\
\hline
\end{tabular}
REDI ${ }^{T M}$.

Se debe regresar a la Tabla 1 para revisar los objetivos de categorización

Según la Tabla 1, el Bloque 3 sin aisladores califica en la categoría PLATA por el porcentaje de pérdida financiera, pero no cumple el requerimiento de tener derivas < $0.5 \%$; por lo tanto no entra dentro de ninguna de las categorías del REDI ${ }^{\mathrm{TM}}$.

El Bloque 3 con aisladores de base triple péndulo de fricción en todas las columnas califica dentro de la categoría ORO, por cumplir el requerimiento de porcentaje de pérdida financiera y derivas máximas. 


\section{CALCULO DEL COSTO DE REPARACIÓN ESTIMADO DE MAMPOSTERÍA EN EL EDIFICIO BUZIOS, USANDO LA CURVA DE FRAGILIDAD DE PACT}

El edificio BUZIOS de gran altura (16 pisos) se ubica al pie de la playa Barbasquillo, entre a la avenida Umiña 3 y calle Umiña 2 en la cuidad de Manta, Provincia de Manabí, dicho edificio fue afectado por el sismo del 16 de abril de 2016, y tuvo mayor afectación en los elementos no estructurales especialmente la mampostería.

Para realizar la validación de las curvas de fragilidad que utiliza el programa PACT, se evalúa la mampostería de dicho edificio, se utiliza los desplazamientos reales post-terremoto para calcular las derivas en cada piso.

Tabla 25 Desplazamientos reales post-terremoto edificio Buzios (Aguiar,

\begin{tabular}{|c|c|c|c|c|}
\hline \multirow[b]{3}{*}{ Piso } & \multirow{2}{*}{\multicolumn{2}{|c|}{ Componente E-W }} & \multicolumn{2}{|c|}{ 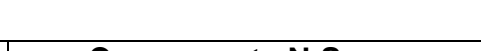 } \\
\hline & & & Compone & N-S \\
\hline & $\begin{array}{l}\text { Sentido } \\
\text { Longitudinal } \\
\text { (cm) }\end{array}$ & $\begin{array}{l}\text { Sentido } \\
\text { Transversal } \\
\text { (cm) }\end{array}$ & $\begin{array}{l}\text { Sentido } \\
\text { Longitudinal } \\
\text { (cm) }\end{array}$ & $\begin{array}{l}\text { Sentido } \\
\text { Transversal } \\
\text { (cm) }\end{array}$ \\
\hline 16 & 23,82 & 35,45 & 13,99 & 22,34 \\
\hline 15 & 22,15 & 30,34 & 12,98 & 18,74 \\
\hline 14 & 20,46 & 28,4 & 11,97 & 17,48 \\
\hline 13 & 18,7 & 26,49 & 10,93 & 16,28 \\
\hline 12 & 16,87 & 24,43 & 9,86 & 15 \\
\hline 11 & 15 & 22,22 & 8,77 & 13,67 \\
\hline 10 & 13,07 & 19,93 & 7,67 & 12,32 \\
\hline 9 & 11,17 & 17,7 & 6,58 & 10,96 \\
\hline 8 & 9,26 & 15,2 & 5,5 & 9,57 \\
\hline 7 & 7,29 & 12,74 & 4,43 & 8,13 \\
\hline 6 & 5,59 & 10,3 & 3,38 & 6,66 \\
\hline 5 & 3,91 & 7,85 & 2,39 & 5,16 \\
\hline 4 & 2,41 & 5,83 & 1,5 & 3,69 \\
\hline 3 & 1,2 & 3,45 & 0,76 & 2,33 \\
\hline 2 & 0,6 & 1,8 & 0,39 & 1,24 \\
\hline 1 & 0,2 & 0,52 & 0,13 & 0,38 \\
\hline
\end{tabular}

Se calcula las derivas en cada piso con cada desplazamiento. De todas las derivas que se calcula, se toma la máxima para la evaluación de la mampostería. 
Tabla 26 Derivas calculadas edificio Buzios

\begin{tabular}{|c|c|c|c|c|c|}
\hline \multirow[b]{2}{*}{ Piso } & \multicolumn{2}{|c|}{ DERIVAS E-W } & \multicolumn{2}{|c|}{ DERIVAS N -S } & \multirow{2}{*}{$\begin{array}{r}\text { DERIV } \\
\text { A MÁX (\%) }\end{array}$} \\
\hline & $(\%)$ Long & $\begin{array}{l}\text { Trans } \\
(\%)\end{array}$ & $(\%)^{\text {Long }}$ & (\%) & \\
\hline 16 & 0,4771 & 1,4600 & 0,2886 & 1,0286 & 1,4600 \\
\hline 15 & 0,4829 & 0,5543 & 0,2886 & 0,3600 & 0,5543 \\
\hline 14 & 0,5029 & 0,5457 & 0,2971 & 0,3429 & 0,5457 \\
\hline 13 & 0,5229 & 0,5886 & 0,3057 & 0,3657 & 0,5886 \\
\hline 12 & 0,5343 & 0,6314 & 0,3114 & 0,3800 & 0,6314 \\
\hline 11 & 0,5514 & 0,6543 & 0,3143 & 0,3857 & 0,6543 \\
\hline 10 & 0,5429 & 0,6371 & 0,3114 & 0,3886 & 0,6371 \\
\hline 9 & 0,5457 & 0,7143 & 0,3086 & 0,3971 & 0,7143 \\
\hline 8 & 0,5629 & 0,7029 & 0,3057 & 0,4114 & 0,7029 \\
\hline 7 & 0,4857 & 0,6971 & 0,3000 & 0,4200 & 0,6971 \\
\hline 6 & 0,4800 & 0,7000 & 0,2829 & 0,4286 & 0,7000 \\
\hline 5 & 0,4286 & 0,5771 & 0,2543 & 0,4200 & 0,5771 \\
\hline 4 & 0,3457 & 0,6800 & 0,2114 & 0,3886 & 0,6800 \\
\hline 3 & 0,1714 & 0,4714 & 0,1057 & 0,3114 & 0,4714 \\
\hline 2 & 0,1143 & 0,3657 & 0,0743 & 0,2457 & 0,3657 \\
\hline 1 & 0,0571 & 0,1486 & 0,0371 & 0,1086 & 0,1486 \\
\hline
\end{tabular}

\section{Descripción del elemento usado}

(FEMA P-58 VOL 3, 2012). Paredes de mampostería: con elementos parcialmente cementados, domina el corte, 4" a 6" (10 a $15 \mathrm{~cm})$ espesor, hasta 12 pies $(3.65 \mathrm{~m})$ de altura. El parámetro de demanda que rige es la deriva de piso.

Estados de daño DS1.- Primera aparición de grandes grietas diagonales, las grietas permanecen cerradas son apenas perceptibles.

Estado de daño DS2.- Grietas diagonales anchas una o más en cada dirección, y el aplastamiento o desprendimiento en la cabeza o pie de pared.
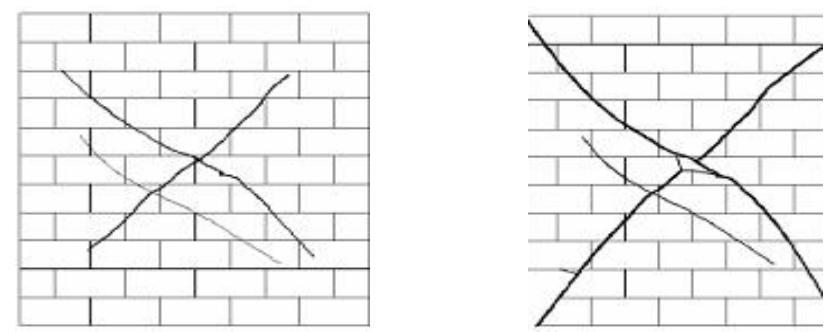

Figura 22 Daños según DS1 izquierda y DS2 a la derecha. (FEMA P-58 VOL 3, 2012) 
Se presenta la curva de fragilidad para mampostería que se obtiene del programa PACT, para la evaluación.

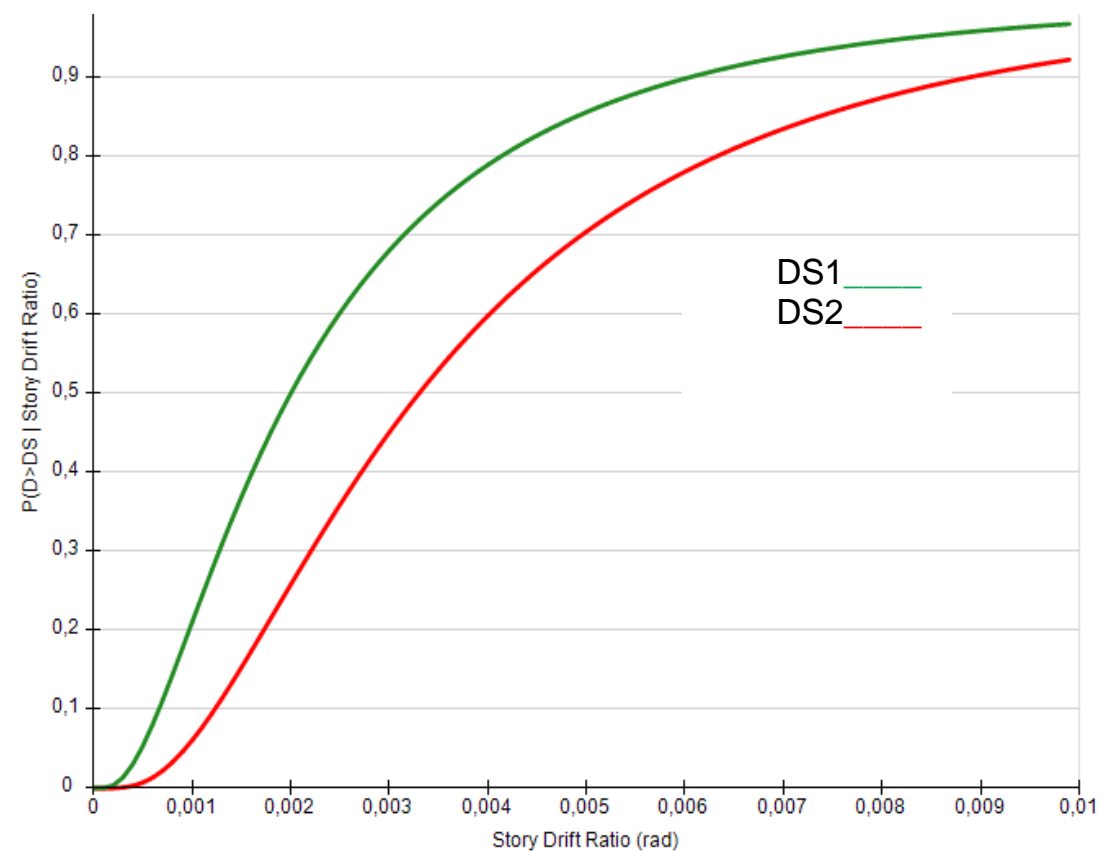

Figura 23 Curva de fragilidad de mampostería (FEMA P-58 VOL 3, 2012)

Con las derivas máximas de piso se ingresa a la curva de fragilidad de mampostería, y se tiene el porcentaje por cada estado de daño. La fórmula que se usa para obtener el costo estimado de reparación es:

$$
\mathrm{C} \mathrm{T}=\text { \%Daño (DS1 o DS2) } * \text { Costo reparación (DS1 o DS2) }
$$

Tabla 27 Porcentajes de daño de mampostería/piso

\begin{tabular}{|c|c|c|c|c|}
\hline \multirow{2}{*}{ Piso } & \multirow{2}{*}{$\begin{array}{c}\text { DERIVA } \\
\text { MÁX (\%) }\end{array}$} & \multicolumn{3}{|c|}{ ESTADOS DE DAÑO } \\
\cline { 3 - 5 } & & NO HAY DAÑO (\%) & DS1 (\%) & DS2 (\%) \\
\hline $\mathbf{1 6}$ & 1,4600 & 0,00 & 0,00 & 100,00 \\
\hline $\mathbf{1 5}$ & 0,5543 & 11,00 & 15,00 & 74,00 \\
\hline $\mathbf{1 4}$ & 0,5457 & 11,00 & 16,00 & 73,00 \\
\hline $\mathbf{1 3}$ & 0,5886 & 10,00 & 14,00 & 76,00 \\
\hline $\mathbf{1 2}$ & 0,6314 & 8,00 & 12,00 & 80,00 \\
\hline $\mathbf{1 1}$ & 0,6543 & 8,00 & 10,00 & 82,00 \\
\hline $\mathbf{1 0}$ & 0,6371 & 9,00 & 10,50 & 80,50 \\
\hline $\mathbf{9}$ & 0,7143 & 7,00 & 10,00 & 83,00 \\
\hline
\end{tabular}




\begin{tabular}{|l|l|c|r|r|}
$\mathbf{8}$ & 0,7029 & 7,00 & 11,00 & 82,00 \\
\hline $\mathbf{7}$ & 0,6971 & 8,00 & 11,00 & 81,00 \\
\hline $\mathbf{6}$ & 0,7000 & 7,00 & 11,00 & 82,00 \\
\hline $\mathbf{5}$ & 0,5771 & 11,00 & 14,00 & 75,00 \\
\hline $\mathbf{4}$ & 0,6800 & 8,00 & 11,00 & 81,00 \\
\hline $\mathbf{3}$ & 0,4714 & 16,00 & 16,00 & 68,00 \\
\hline $\mathbf{2}$ & 0,3657 & 25,00 & 20,00 & 55,00 \\
\hline $\mathbf{1}$ & 0,1486 & 68,00 & 20,00 & 12,00 \\
\hline
\end{tabular}

El costo DS2/M2 se obtiene del desglose de precios de Bloque + enlucido $=36.00$ USD, Pintura $=10.30$ USD y imprevistos $=3.70$ USD aproximadamente, se obtiene un valor total para DS2 = 50.00 USD; y para DS1 se toma el $50 \%$ de DS2. (PARANA, 2016).

Tabla 28 Costos de reparación de mampostería/piso

\begin{tabular}{|c|c|c|c|c|c|c|}
\hline \multirow{2}{*}{ Piso } & Costo DS1/M2 & Costo DS2/M2 & & Área & & \\
\cline { 2 - 7 } & $\mathbf{2 5}$ & $\mathbf{5 0}$ & Costo/PISO/M2 & $\begin{array}{c}\text { Mampostería } \\
\text { (m2) }\end{array}$ & Costo/PISO & H Piso (m) \\
\hline $\mathbf{1 6}$ & 0,00 & 50,00 & 50,00 & 244,34 & 12216,75 & 56,00 \\
\hline $\mathbf{1 5}$ & 3,75 & 37,00 & 40,75 & 244,34 & 9956,65 & 52,50 \\
\hline $\mathbf{1 4}$ & 4,00 & 36,50 & 40,50 & 814,45 & 32985,23 & 49,00 \\
\hline $\mathbf{1 3}$ & 3,50 & 38,00 & 41,50 & 814,45 & 33799,68 & 45,50 \\
\hline $\mathbf{1 2}$ & 3,00 & 40,00 & 43,00 & 814,45 & 35021,35 & 42,00 \\
\hline $\mathbf{1 1}$ & 2,50 & 41,00 & 43,50 & 814,45 & 35428,58 & 38,50 \\
\hline $\mathbf{1 0}$ & 2,63 & 40,25 & 42,88 & 814,45 & 34919,54 & 35,00 \\
\hline $\mathbf{9}$ & 2,50 & 41,50 & 44,00 & 814,45 & 35835,80 & 31,50 \\
\hline $\mathbf{8}$ & 2,75 & 41,00 & 43,75 & 814,45 & 35632,19 & 28,00 \\
\hline $\mathbf{7}$ & 2,75 & 40,50 & 43,25 & 814,45 & 35224,96 & 24,50 \\
\hline $\mathbf{6}$ & 2,75 & 41,00 & 43,75 & 814,45 & 35632,19 & 21,00 \\
\hline $\mathbf{5}$ & 3,50 & 37,50 & 41,00 & 814,45 & 33392,45 & 17,50 \\
\hline $\mathbf{4}$ & 2,75 & 40,50 & 43,25 & 814,45 & 35224,96 & 14,00 \\
\hline $\mathbf{3}$ & 4,00 & 34,00 & 38,00 & 325,78 & 12379,64 & 10,50 \\
\hline $\mathbf{2}$ & 5,00 & 27,50 & 32,50 & 325,78 & 10587,85 & 7,00 \\
\hline $\mathbf{1}$ & 5,00 & 6,00 & 11,00 & 325,78 & 3583,58 & 3,5 \\
\hline
\end{tabular}

Los tres primeros pisos son parqueaderos y cuartos de máquinas, los dos últimos pisos son terraza y área comunal del edificio; por tal motivo su área de mampostería es menor. 


\section{RESULTADOS Y DISCUSIÓN EDIFICIO BUZIOS}

A continuación se presentan los resultados de la evaluación que se realiza al edificio Buzios con la curva de fragilidad del programa PACT.

Tabla 29 Resumen costos y porcentaje de reparación de mampostería del Edificio Buzios

\begin{tabular}{|c|c|c|}
\hline $\begin{array}{c}\text { Área Total } \\
\text { Mampostería }\end{array}$ & 10424,96 & m2 \\
\hline $\begin{array}{c}\text { Costo } \\
\text { Mampostería/m2 }\end{array}$ & $\$ 50,00$ & USD \\
\hline $\begin{array}{c}\text { Costo } \\
\text { Mampostería } \\
\text { Edificio Buzios }\end{array}$ & $\$ 521.248,00$ & USD \\
\hline $\begin{array}{c}\text { Costo Reparación } \\
\text { Mampostería }\end{array}$ & $\$ 431.821,39$ & USD \\
\hline $\begin{array}{c}\text { Porcentaje de } \\
\text { Reparación de } \\
\text { Mampostería }\end{array}$ & 82,84 & $\%$ \\
\hline
\end{tabular}

Como se observa en la tabla, la mampostería se afecta un $82.84 \%$, lo que evidencia que los elementos no estructurales son más vulnerables ante eventos sísmicos.

Tabla 30 Resumen costos y porcentaje de reparación del Edificio Buzios

\begin{tabular}{|c|c|c|}
\hline Área/piso & 732,68 & $\mathbf{~ m 2}$ \\
\hline Área Total & 11722,81 & $\mathbf{~ m 2}$ \\
\hline $\begin{array}{c}\text { Costo } \\
\text { Construcción/m2 }\end{array}$ & $\$ 650,00$ & USD \\
\hline $\begin{array}{c}\text { Costo Total Edificio } \\
\text { Buzios }\end{array}$ & $\$ 7.619 .826,24$ & USD \\
\hline $\begin{array}{c}\text { Costo Reparación } \\
\text { Porcentaje de } \\
\text { Reparación Total }\end{array}$ & $5431.821,39$ & USD \\
\hline
\end{tabular}

El edificio Buzios tiene derivas mayores al $0.5 \%$, lo que impide que entre en la calificación REDI ${ }^{\mathrm{TM}}$, pero según su porcentaje de pérdida financiera cumple para tener una calificación PLATA. 
El costo de reparación real más el reforzamiento del edificio Buzios es USD 1'300.000; el costo de reparación solamente de mampostería es $35 \%$ del costo total, es decir USD 455.000. El costo de reparación estimado de mampostería obtenido con las curvas del programa PACT es USD 431.821,39, comparando los dos valores se tiene una diferencia de USD 23.178.61, que representa un error de 5.09\%; con lo cual se puede validar las curvas de fragilidad que utiliza el programa PACT en las evaluaciones.

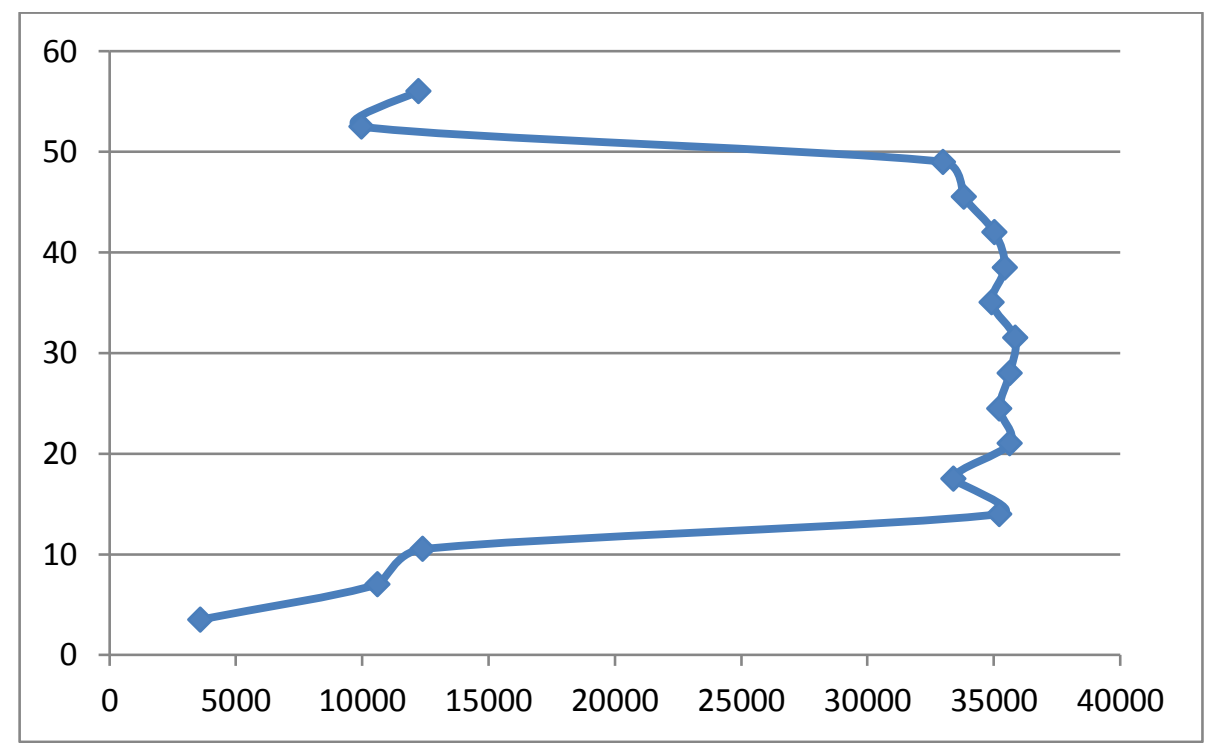

Figura 24 Curva con los costos de reparación de mampostería por piso

\section{COMENTARIOS Y CONCLUSIONES}

Se calificó al Bloque 3 con aisladores de triple péndulo de fricción del Nuevo Centro de Investigaciones y Postgrados de la Universidad de las Fuerzas Armadas ESPE, en la categoría Oro según REDI ${ }^{\mathrm{TM}}$, por tener una pérdida financiera $<5 \%$ del costo total, y derivas menores al $0,5 \%$.

El Bloque 3 sin aisladores del Nuevo Centro de Investigaciones y Postgrados de la Universidad de las Fuerzas Armadas ESPE, calificó dentro de la categoría Plata por tener una pérdida financiera < 10\% del costo total según $\mathrm{REDI}^{\mathrm{TM}}$, pero no cumplió con el requerimiento de derivas menores al $0,5 \%$, por lo tanto no entra en ninguna categoría.

El edifico Buzios no entra en la categorización REDI ${ }^{\mathrm{TM}}$ por tener derivas mayores al 0,5\%. El cálculo del costo de reparación de la mampostería del edificio Buzios sirvió para validar las curvas de fragilidad del programa PACT, ya que se obtuvo un valor calculado similar al valor real del costo de reparación.

Un factor importante para que las estructuras entren al sistema de calificación de costos REDI $I^{\mathrm{TM}}$ es la deriva, la cual es un indicador fundamental para que existan menores daños y consecuentemente menores pérdidas financieras. 
Las estructuras a pesar de cumplir con el valor de $2 \%$ de deriva que indica el NEC 14, sufren una pérdida financiera alta, de ahí la necesidad que el diseño de edificaciones vaya más allá de los valores mínimos propuestos en la norma.

\section{REFERENCIAS}

1. Aguiar, Roberto. (2012). Dinámica de estructuras con CEINCI-LAB. Universidad de las Fuerzas Armadas-ESPE.

2. Aguiar R, Pazmiño E. (2016). "Detalles constructivos debido a los aisladores sísmicos en las construcciones de la Universidad de las Fuerzas Armadas ESPE" Revista Internacional de Ingeniería de Estructuras, 21, (2), 217-256. Disponible en: http//www.riie.espe.edu.ec.

3. Aguiar, Roberto (2016). Análisis espacial espectral del edificio Buzios en Manta.

4. FEMA P-58 (Septiembre 2012). Seismic Performance Assessment of Buildings, Methodology Volumen 1 FEDERAL EMERGENCY MANAGEMENT AGENCY, Washintong, D.C.

5. FEMA P-58 (Septiembre 2012). Seismic Performance Assessment of Buildings, Implementation Volumen 2 FEDERAL EMERGENCY MANAGEMENT AGENCY, Washintong, D.C.

6. FEMA P-58 (Septiembre 2012). Seismic Performance Assessment of Buildings, Volumen 3 Supporting Electronic Materials and Background Documentation. Volumen3 FEDERAL EMERGENCY MANAGEMENT AGENCY, Washintong, D.C.

7. Ibrahim Almufti and Michael Willford (October 2013) REDi ${ }^{\mathrm{TM}}$ Rating System - Resilience-based Earthquake Design Initiative for the Next Generation of Buildings.

8. Mora D. y Aguiar R. (Diciembre 2015) Comportamiento de Estructuras de Acero Con Y Sin Disipadores de Energía Tipo TADAS, Ubicadas en la Ciudad de Quito, por el Método del Espectro de Capacidad.

9. NEC-15 (Agosto 2014) Norma Ecuatoriana de la Construcción, SE-DS, Curva de Peligro Sísmico. Curva de Peligrosidad de Quito.

10. PARANA (AR) "Costos de construcción Julio 2016". Recuperado de: http://www.solucionesespeciales.net/Inmobiliaria/Costos.aspx 